Network Working Group

Request for Comments: 1513

Updates: 1271
S. Waldbusser

Carnegie Mellon University

September 1993

\title{
Token Ring Extensions to the Remote Network Monitoring MIB
}

Status of this Memo

This RFC specifies an Internet standards track protocol for the Internet community, and requests discussion and suggestions for improvements. Please refer to the current edition of the "Internet Official Protocol standards" for the standardization state and status of this protocol. Distribution of this memo is unlimited.

Abstract

This memo defines extensions to the Remote Network Monitoring MIB for managing 802.5 Token Ring networks.

The Remote Network Monitoring MIB, RFC 1271, defines a framework for remote monitoring functions implemented on a network probe. That MIB defines objects broken down into nine functional groups. Some of those functional groups, the statistics and the history groups, have a view of the data-link layer that is specific to the media type and require specific objects to be defined for each media type. RFC 1271 defined those specific objects necessary for Ethernet. This companion memo defines those specific objects necessary for Token Ring LANs.

In addition, this memo defines some additional monitoring functions specifically for Token Ring. These are defined in the Ring station Group, the Ring Station Order Group, the Ring Station Configuration Group, and the Source Routing Statistics Group.

Table of Contents

1. The Network Management Framework ................ 2

2. Guidelines for implementing RFC1271 objects on a

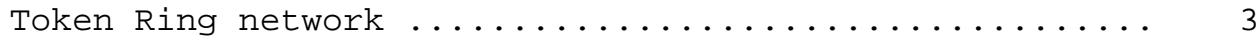

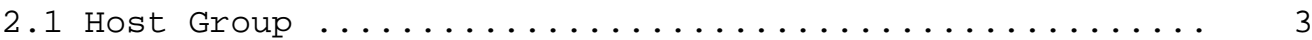

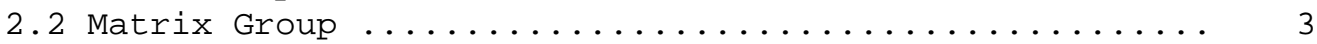

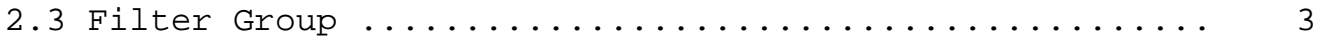

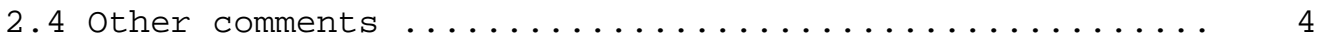

3. Overview of the RMON Token Ring Extensions MIB ...... 4

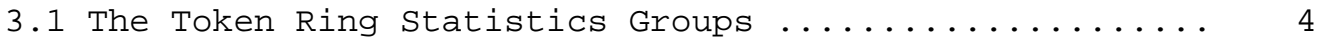

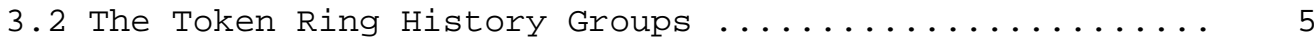

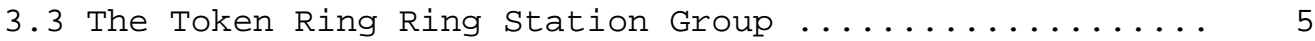


3.4 The Token Ring Ring Station Order Group ................... 5



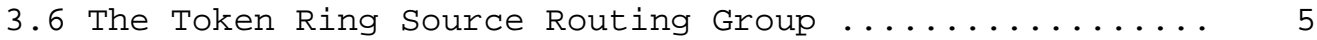

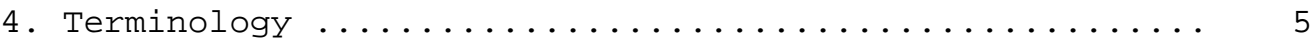

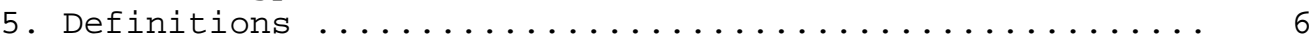

5.1 The Token Ring Mac-Layer Statistics Group .......... 6

5.2 The Token Ring Promiscuous Statistics Group ........ 14



5.4 The Token Ring Promiscuous History Group .......... 27

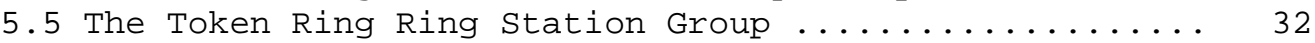

5.6 The Token Ring Ring Station Order Group ........... 41



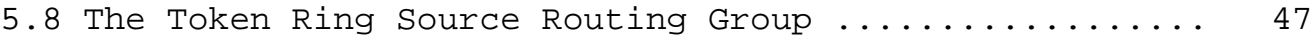

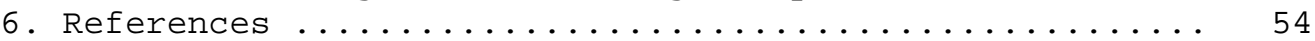

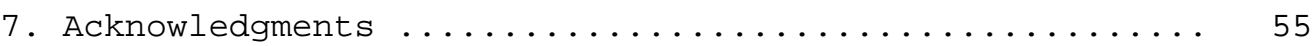

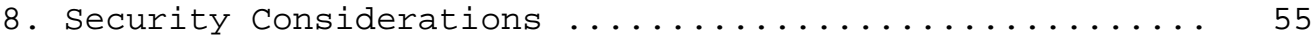

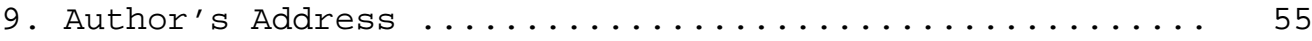

1. The Network Management Framework

The Internet-standard Network Management Framework consists of three components. They are:

STD 16, RFC 1155 [1] which defines the SMI, the mechanisms used for describing and naming objects for the purpose of management. STD 16, RFC 1212 [2] defines a more concise description mechanism, which is wholly consistent with the SMI.

STD 17, RFC 1213 [3] which defines MIB-II, the core set of managed objects for the Internet suite of protocols.

STD 15, RFC 1157 [4] which defines the SNMP, the protocol used for network access to managed objects.

The Framework permits new objects to be defined for the purpose of experimentation and evaluation.

Managed objects are accessed via a virtual information store, termed the Management Information Base or MIB. Within a given MIB module, objects are defined using STD 16, RFC 1212's OBJECT-TYPE macro. At a minimum, each object has a name, a syntax, an access-level, and an implementation-status.

The name is an object identifier, an administratively assigned name, which specifies an object type. The object type together with an object instance serves to uniquely identify a specific instantiation of the object. For human convenience, we often use a textual string, termed the object descriptor, to also refer to the object type. 
The syntax of an object type defines the abstract data structure corresponding to that object type. The ASN.1[5] language is used for this purpose. However, STD 16, RFC 1155 purposely restricts the ASN.1 constructs which may be used. These restrictions are explicitly made for simplicity.

The access-level of an object type defines whether it makes "protocol sense" to read and/or write the value of an instance of the object type. (This access-level is independent of any administrative authorization policy.)

The implementation-status of an object type indicates whether the object is mandatory, optional, obsolete, or deprecated.

2. Guidelines for implementing RFC1271 objects on a Token Ring network

Wherever a MacAddress is to be used in this MIB the source routing bit is stripped off. The resulting address will be consistently valid for all packets sent by a particular node.

\subsection{Host Group}

Only Token Ring isolating errors will increment the error counter for a particular hostentry. The isolating errors are: LineErrors, Bursterrors, ACErrors, InternalErrors, and AbortErrors. ACErrors will increment the error counter only for the nearest upstream neighbor of the station reporting the error. LineErrors and Bursterrors will increment the error counters for the station reporting the error and its neighbor upstream neighbor. InternalErrors and AbortErrors will increment the error counter for the station reporting the error only. In addition, congestionErrors will also be counted for each hostEntry. These errors will be incremented in the host entry of the station that reports the errors in an error report frame.

The hostoutPkts and hostoutoctets counters shall not be incremented for packets with errors.

\subsection{Matrix Group}

Error counters are never incremented.

\subsection{Filter Group}

The following conditions make up the status bitmask for token ring networks: 


\section{Error}

First packet after some packets were dropped Packet with the Frame Copied Bit set

Packet with the Address Recognized Bit set

For the purpose of the packet match algorithm, the filters assume a 32 byte RIF field. Thus, when matching, the filter is compared to the packet starting at the AC byte of the packet, until the end of the RIF field; then the unused RIF bytes in the filter are skipped and matching proceeds from that point. Any filter "care" bits in the RIF that don't correspond to bytes in the input packet will cause the filter to fail.

\subsection{Other comments}

Because soft error report packets may be sent with assured delivery, some errors may be accidently reported twice on devices that perform the RMON function promiscuously.

\section{Overview of the RMON Token Ring Extensions MIB}

The Remote Network Monitoring MIB, RFC 1271, defines a framework for remote monitoring functions implemented on a network probe. That MIB defines objects broken down into nine functional groups. Some of those functional groups, the statistics and the history groups, have a view of the data-link layer that is specific to the media type and require specific objects to be defined for each media type. RFC 1271 defined those specific objects necessary for Ethernet. This MIB defines contains four groups that define those specific objects necessary for Token Ring LANs.

In addition, this memo defines some additional monitoring functions specifically for Token Ring. These are defined in the Ring station Group, the Ring Station Order Group, the Ring Station Configuration Group, and the Source Routing Statistics Group.

\subsection{The Token Ring Statistics Groups}

The Token Ring statistics groups contain current utilization and error statistics. The statistics are broken down into two groups, the Token Ring Mac-Layer Statistics Group and the Token Ring Promiscuous Statistics Group. The Token Ring Mac-Layer Statistics Group collects information from Mac Layer, including error reports for the ring and ring utilization of the Mac Layer. The Token Ring Promiscuous Statistics Group collects utilization statistics from data packets collected promiscuously. 


\subsection{The Token Ring History Groups}

The Token Ring History Groups contain historical utilization and error statistics. The statistics are broken down into two groups, the Token Ring Mac-Layer History Group and the Token Ring Promiscuous History Group. The Token Ring Mac-Layer History Group collects information from Mac Layer, including error reports for the ring and ring utilization of the Mac Layer. The Token Ring Promiscuous History Group collects utilization statistics from data packets collected promiscuously.

\subsection{The Token Ring Ring Station Group}

The Token Ring Ring Station Group contains statistics and status information associated with each Token Ring station on the local ring. In addition, this group provides status information for each ring being monitored.

\subsection{The Token Ring Ring Station Order Group}

The Token Ring Ring Station Order Group provides the order of the stations on monitored rings.

\subsection{The Token Ring Ring Station Config Group}

The Token Ring Ring Station Config Group manages token ring stations through active means. Any station on a monitored ring may be removed or have configuration information downloaded from it.

\subsection{The Token Ring Source Routing Group}

The Token Ring Source Routing Group contains utilization statistics derived from source routing information optionally present in token ring packets.

4. Terminology

The 802.5 specification [7] defines the term "good frame" as a frame that is bounded by a valid SD and ED, is an integral number of octets in length, is composed of only 0 and 1 bits between the $S D$ and the ED, has the FF bits of the GC field equal to 00 or 01 , has a valid FCS, and has a minimum of 18 octets between the SD and the ED. This document will use the term "good frame" in the same manner. 
5. Definitions

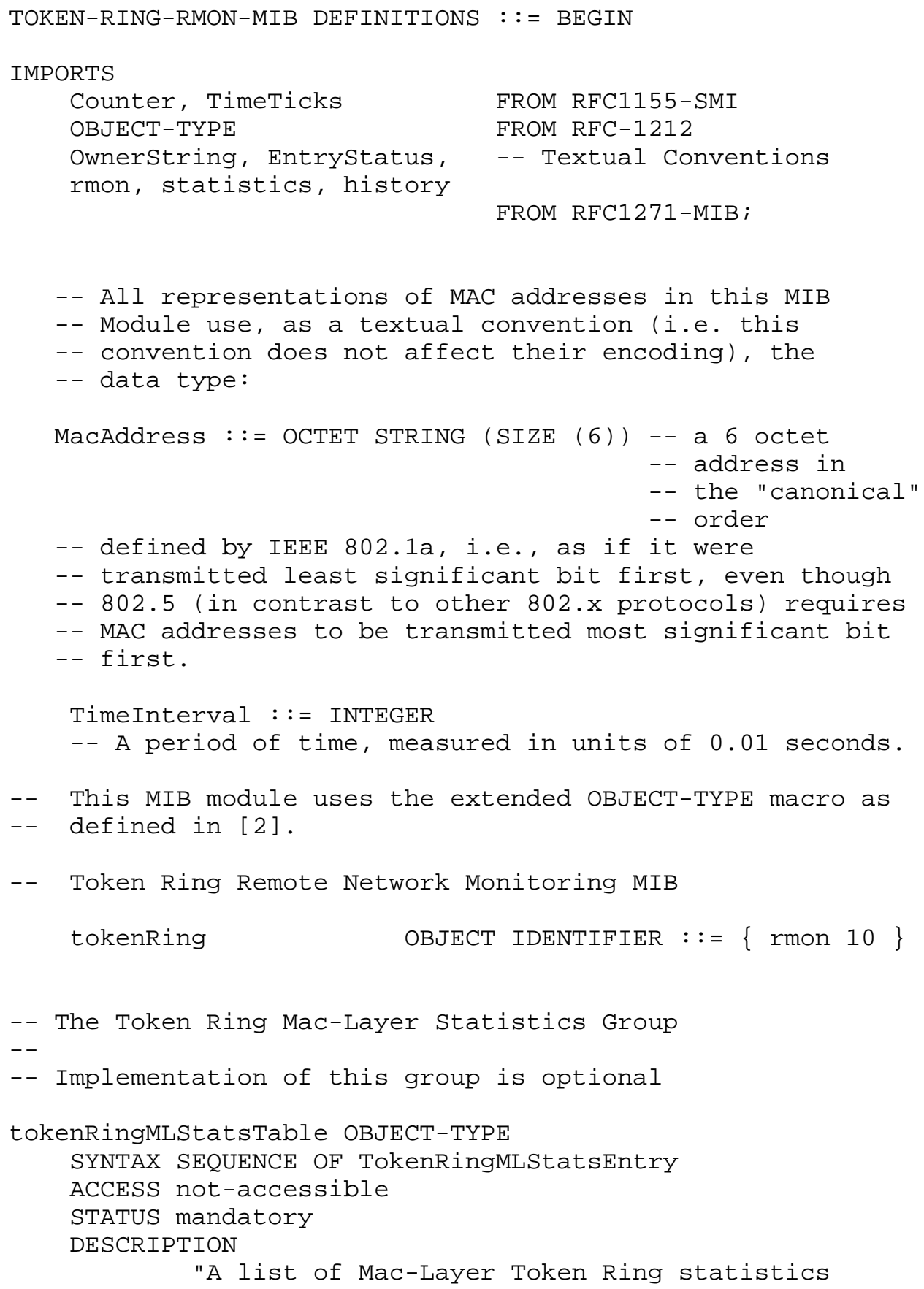

"A list of Mac-Layer Token Ring statistics 


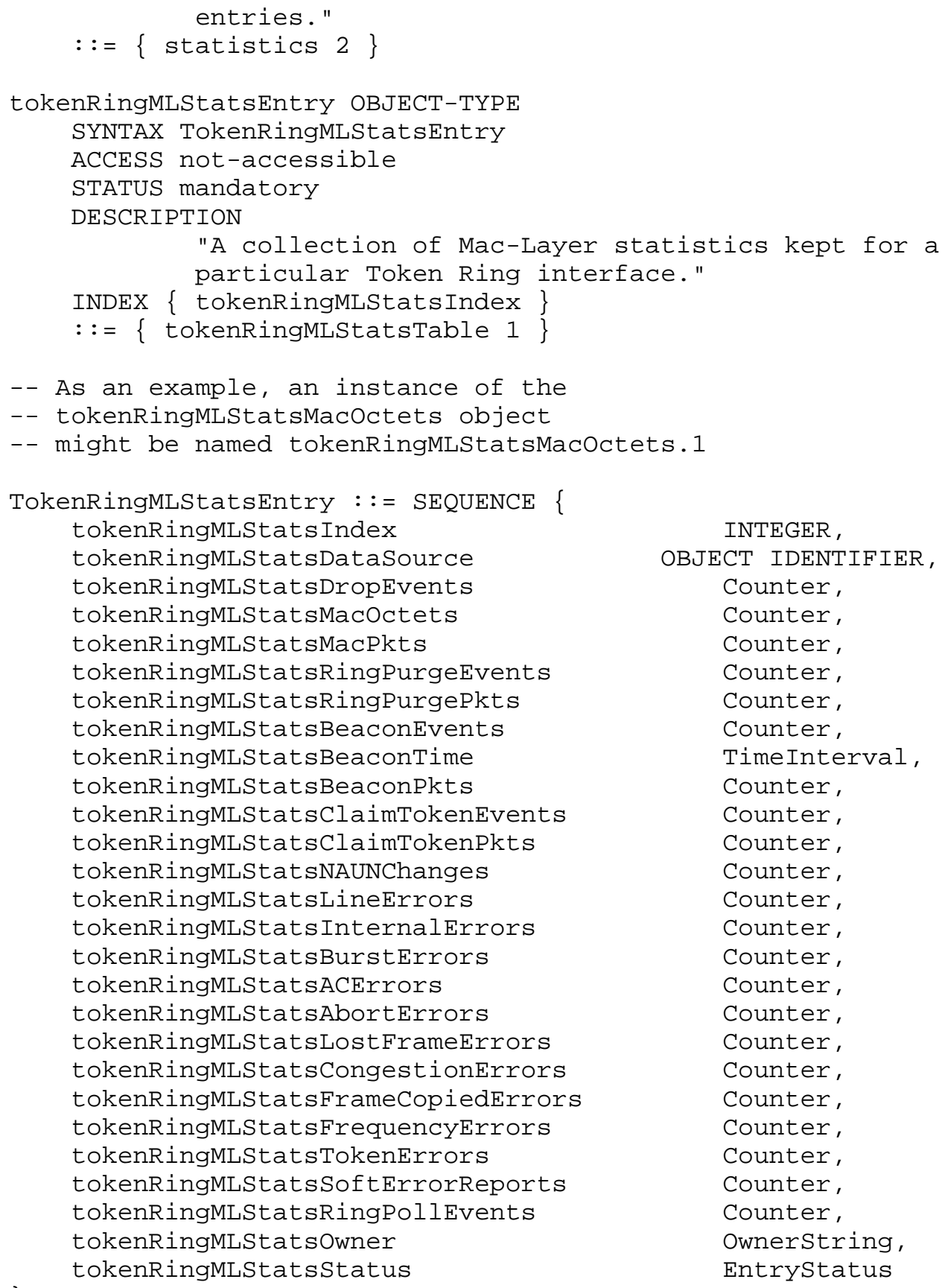




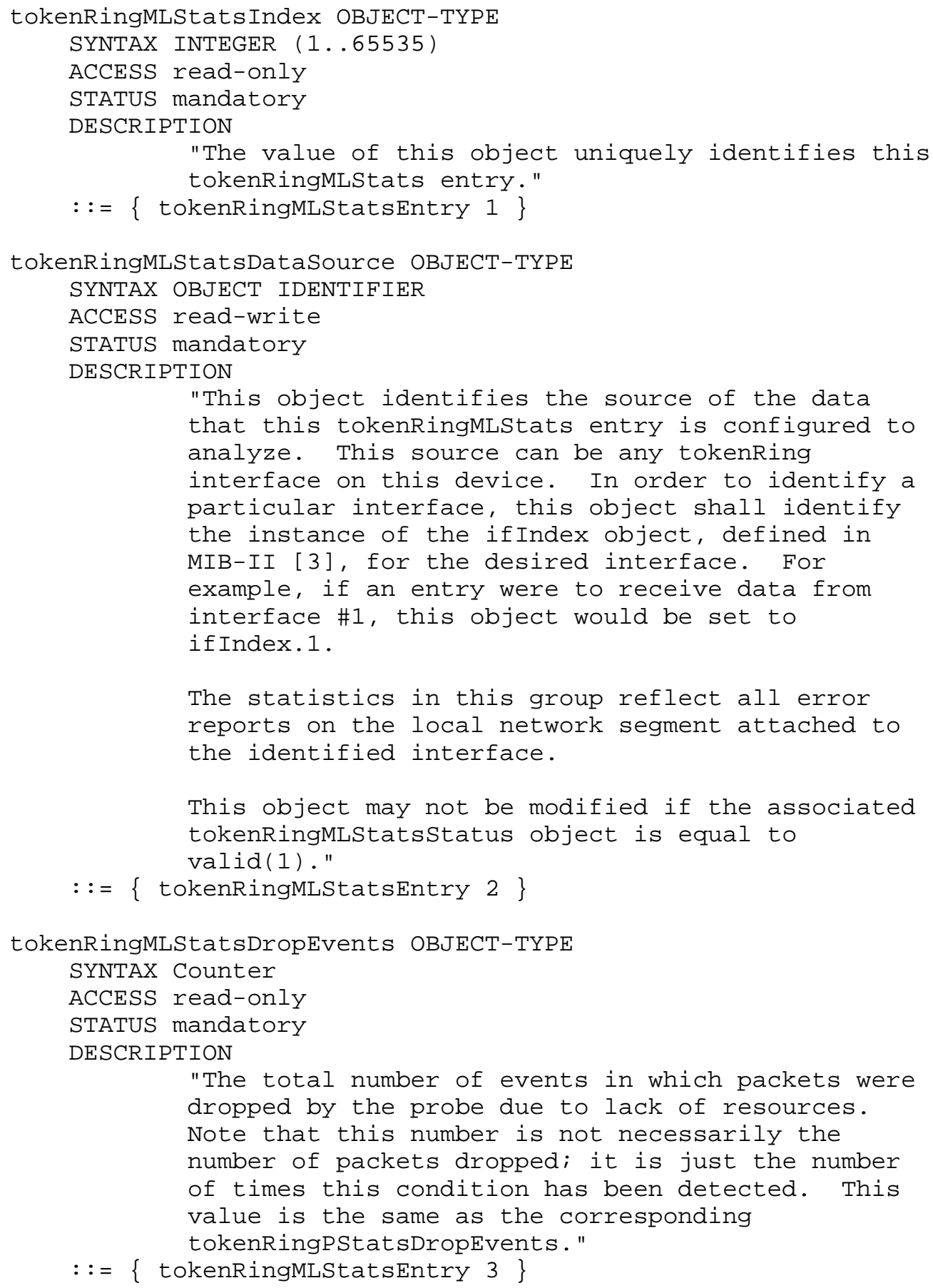

Waldbusser 


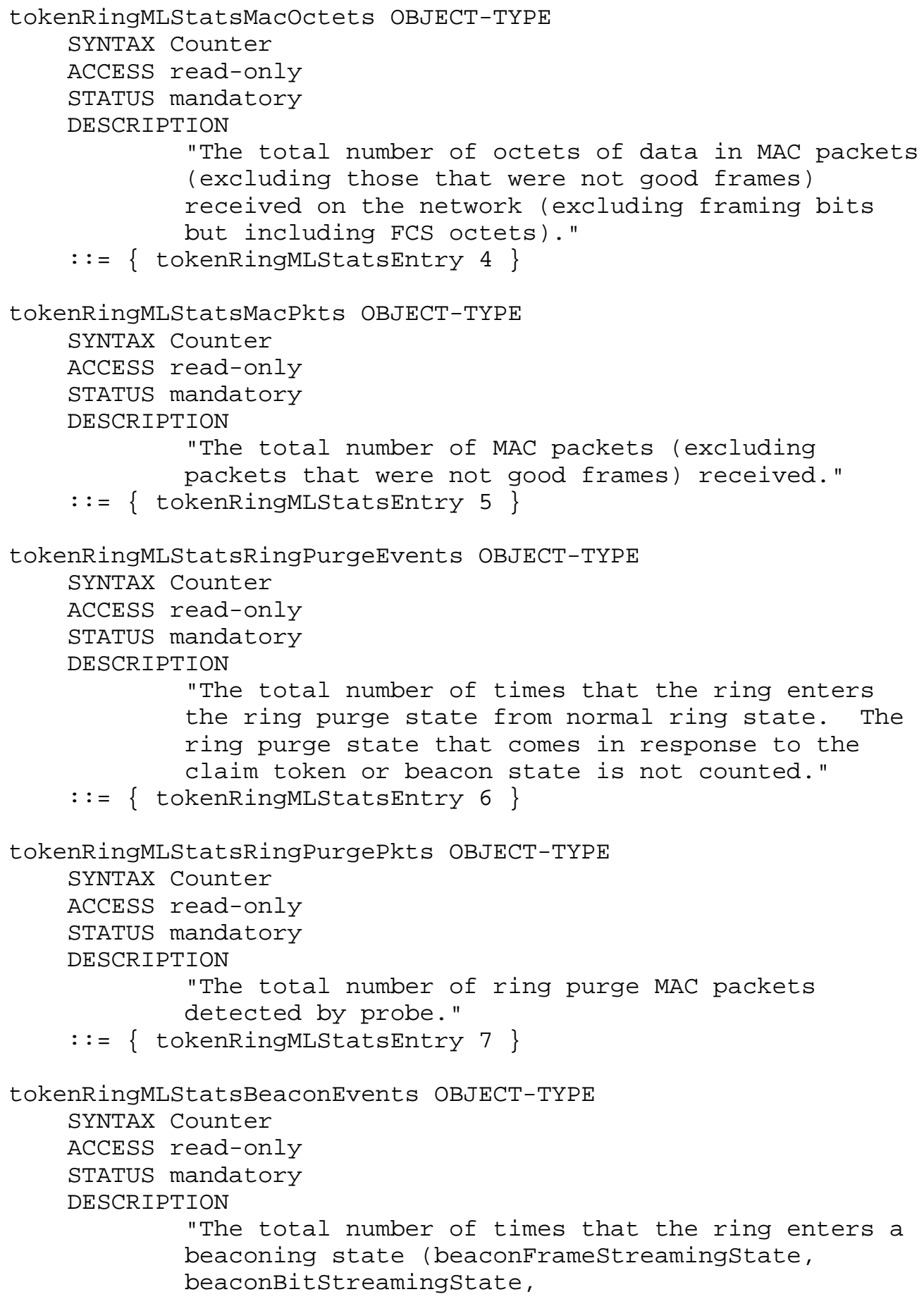




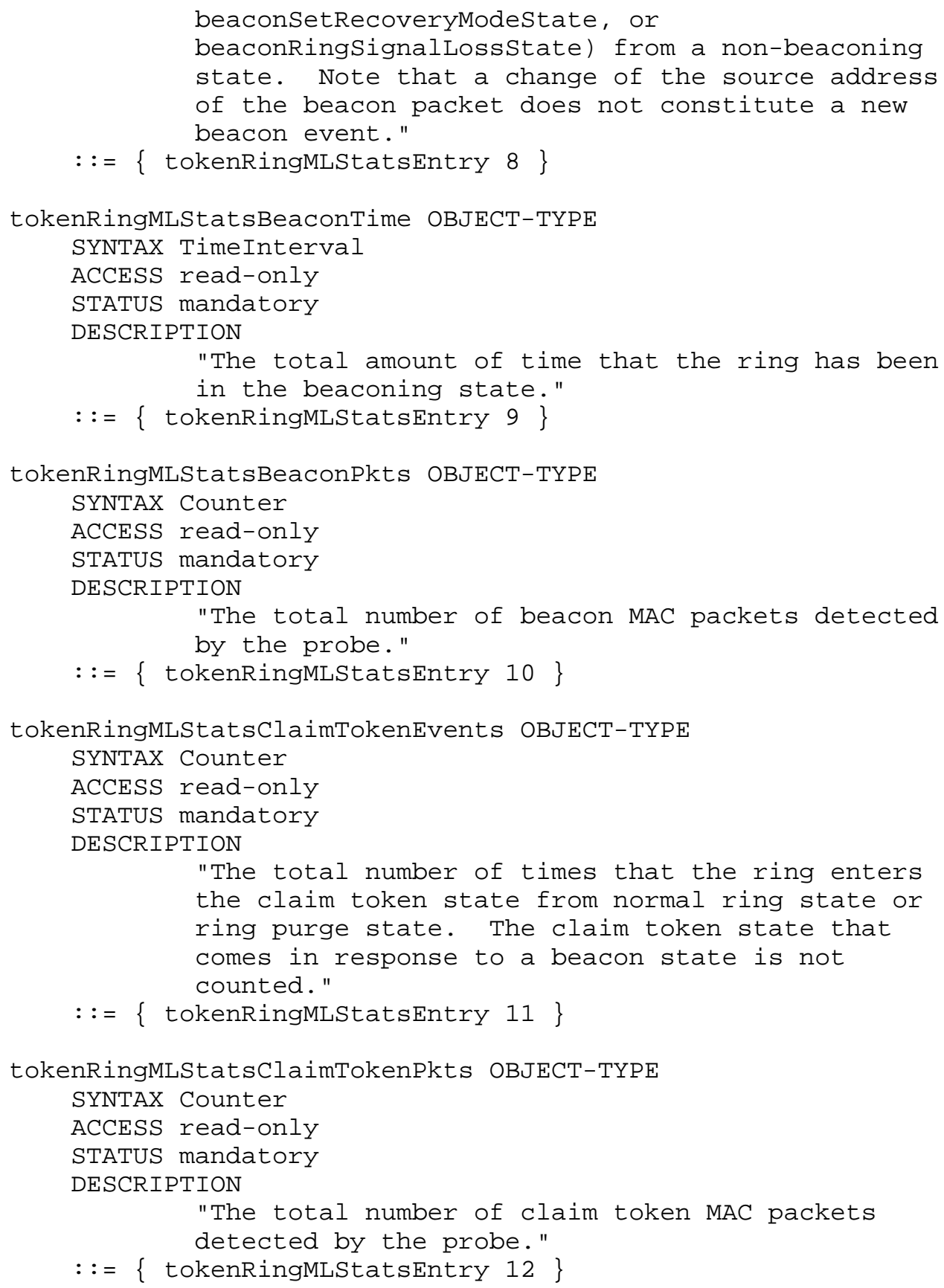









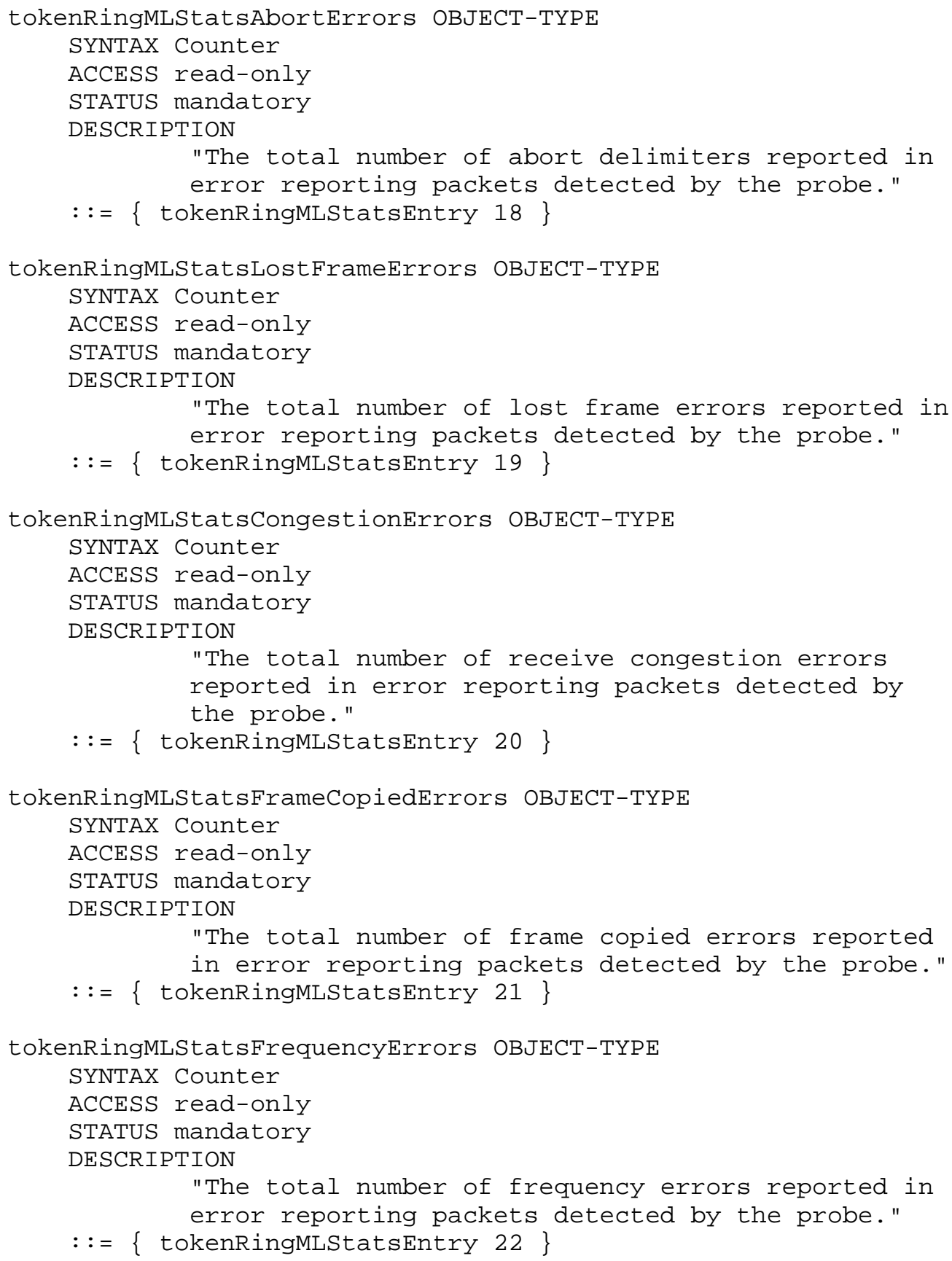




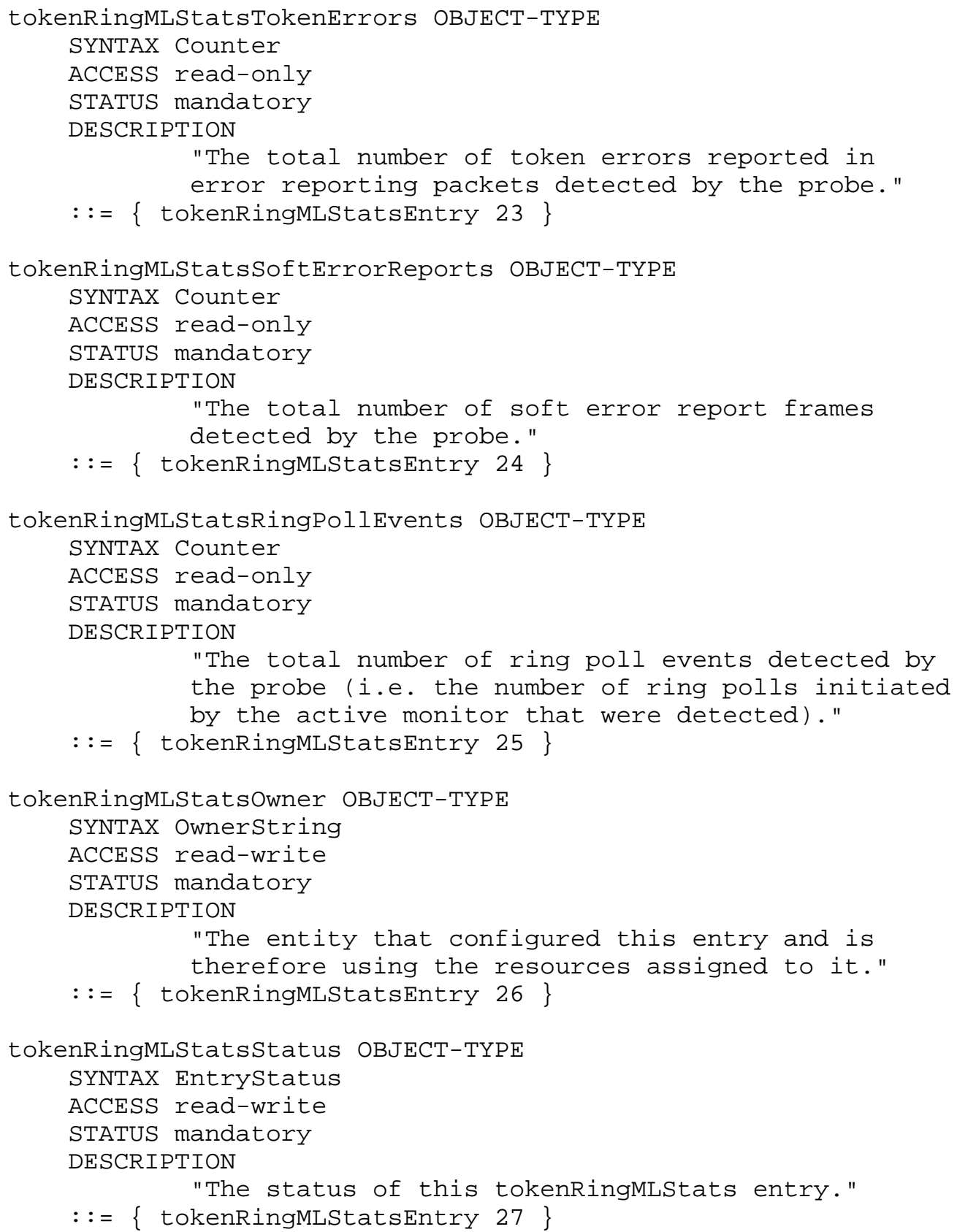




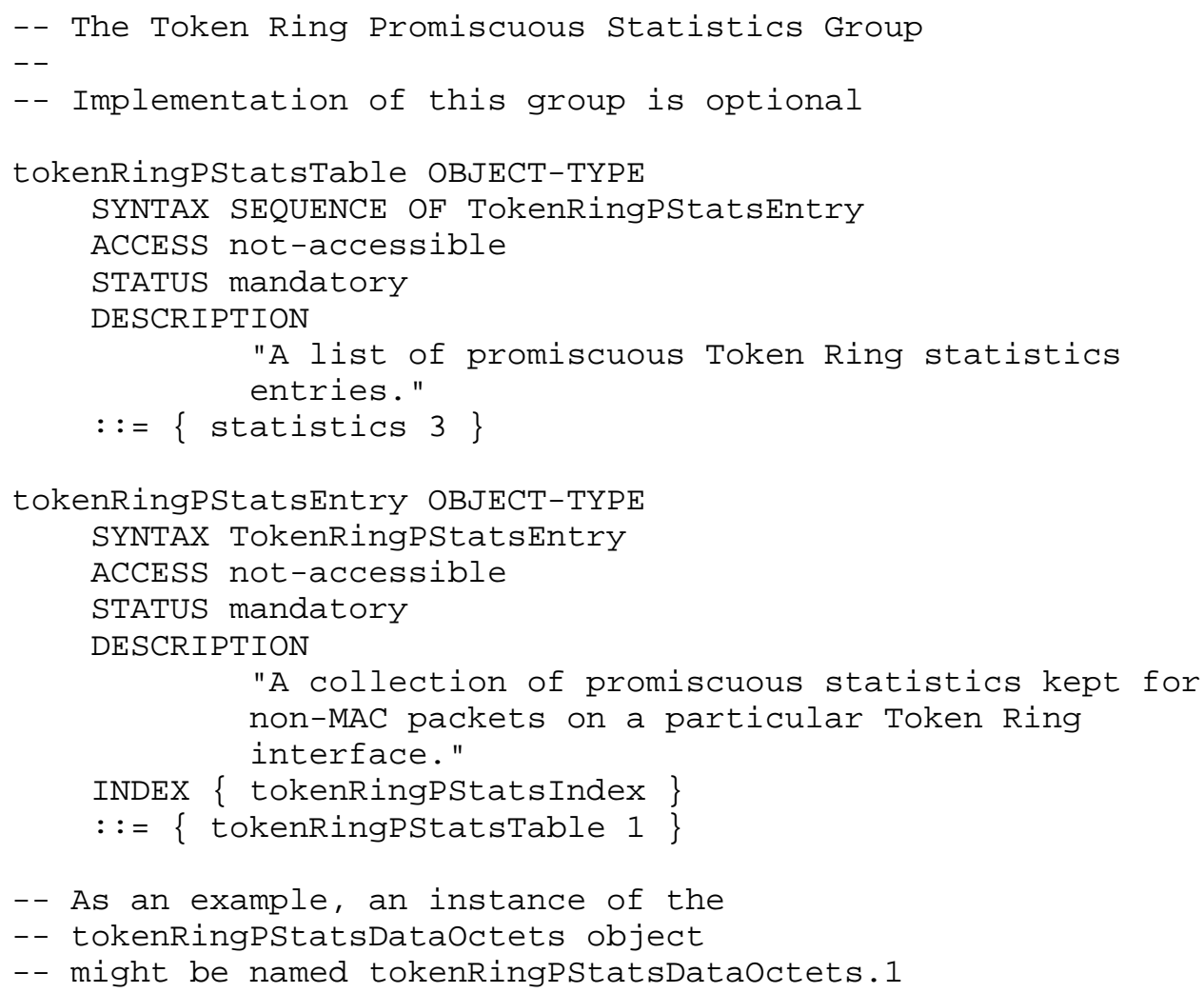


\}

tokenRingPStatsIndex OBJECT-TYPE

SYNTAX INTEGER (1..65535)

ACCESS read-only

STATUS mandatory

DESCRIPTION

"The value of this object uniquely identifies this tokenRingPStats entry."

$::=\{$ tokenRingPStatsEntry 1$\}$

tokenRingPStatsDataSource OBJECT-TYPE

SYNTAX OBJECT IDENTIFIER

ACCESS read-write

STATUS mandatory

DESCRIPTION

"This object identifies the source of the data

that this tokenRingPstats entry is configured to

analyze. This source can be any tokenRing

interface on this device. In order to identify a

particular interface, this object shall identify

the instance of the ifIndex object, defined in

MIB-II [3], for the desired interface. For

example, if an entry were to receive data from

interface \#1, this object would be set to

ifIndex. 1 .

The statistics in this group reflect all non-MAC packets on the local network segment attached to the identified interface.

This object may not be modified if the associated tokenRingPStatsstatus object is equal to valid(1)."

$::=\{$ tokenRingPStatsEntry 2$\}$

tokenRingPStatsDropEvents OBJECT-TYPE

SYNTAX Counter

ACCESS read-only

STATUS mandatory

DESCRIPTION

"The total number of events in which packets were dropped by the probe due to lack of resources. Note that this number is not necessarily the number of packets dropped; it is just the number of times this condition has been detected. This value is the same as the corresponding tokenRingMLStatsDropEvents" 


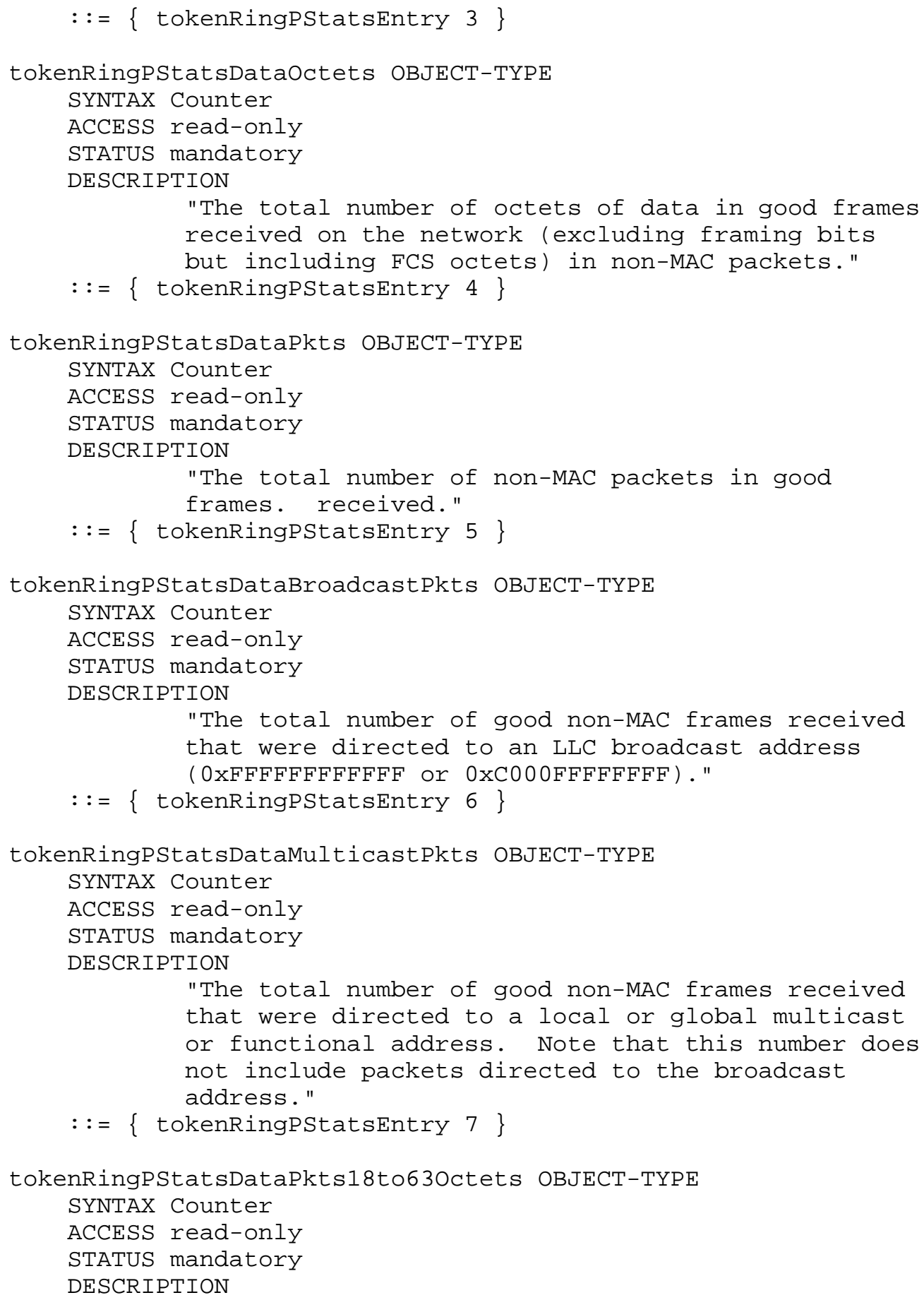




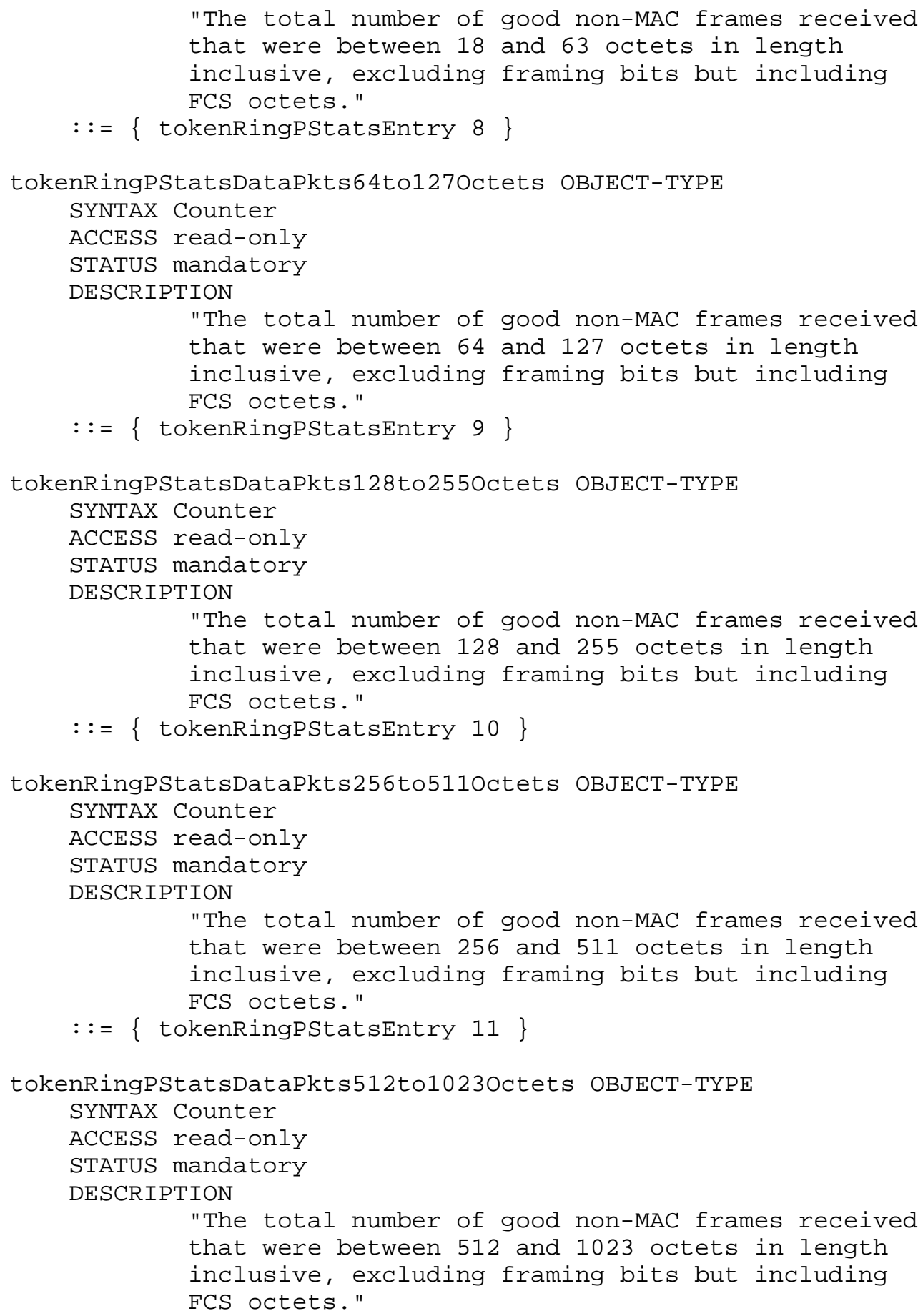




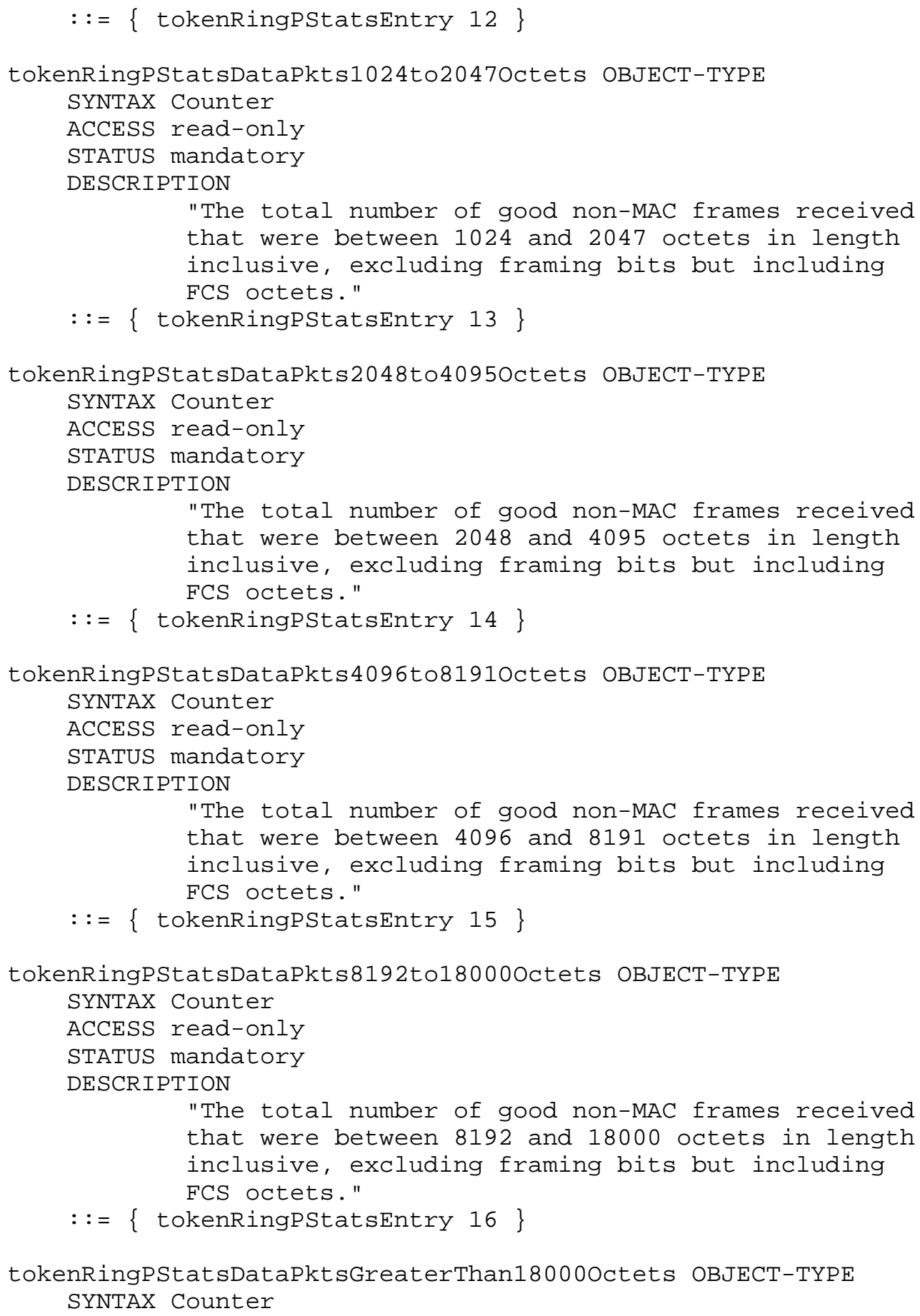




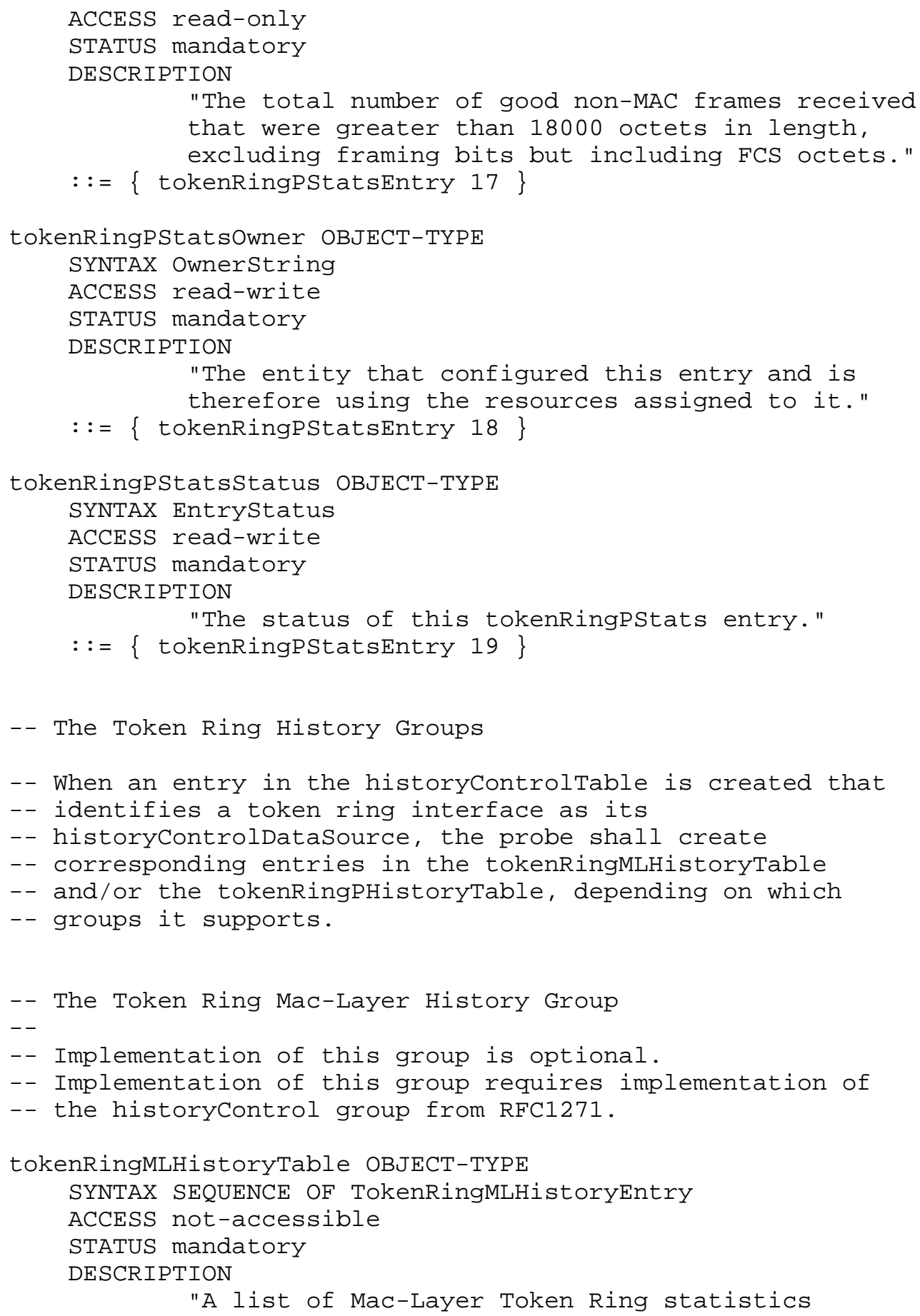

"A list of Mac-Layer Token Ring statistics 


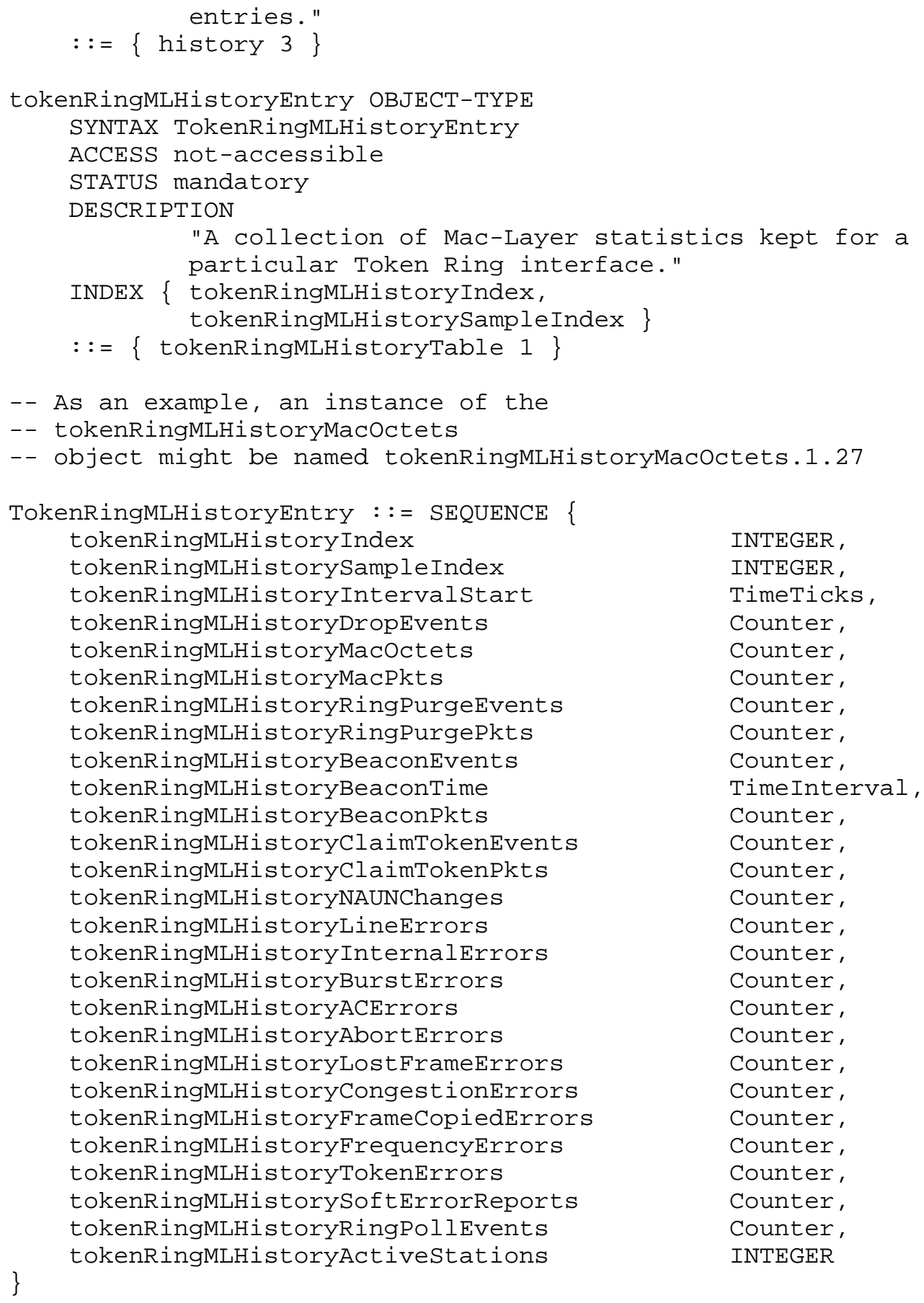

INTEGER, INTEGER, TimeTicks, Counter, Counter, Counter, Counter, Counter, Counter, TimeInterval, Counter, Counter, Counter, Counter, Counter, Counter, Counter, Counter, Counter, Counter, Counter, Counter, Counter, Counter, Counter, Counter, INTEGER 


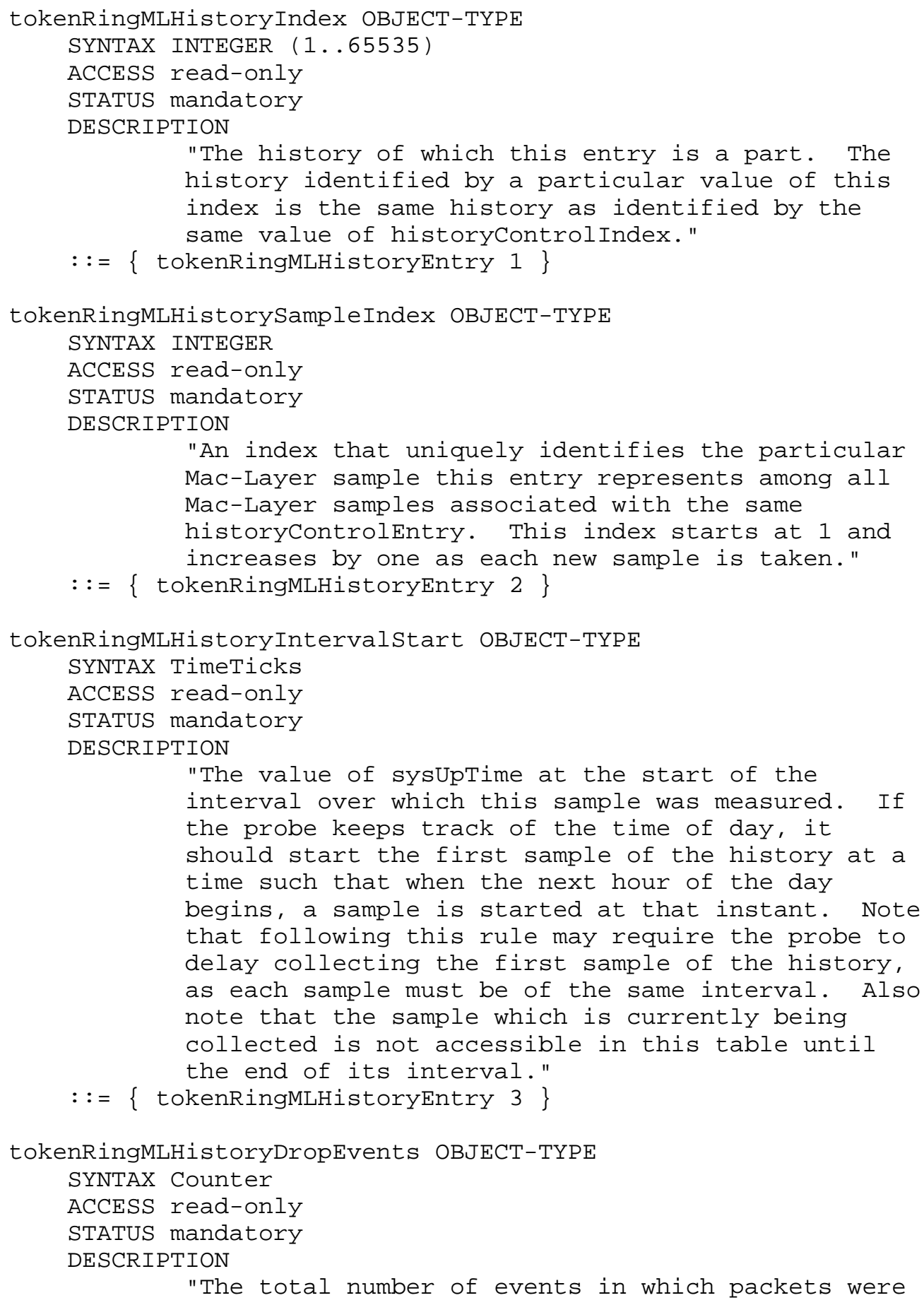

"The total number of events in which packets were 


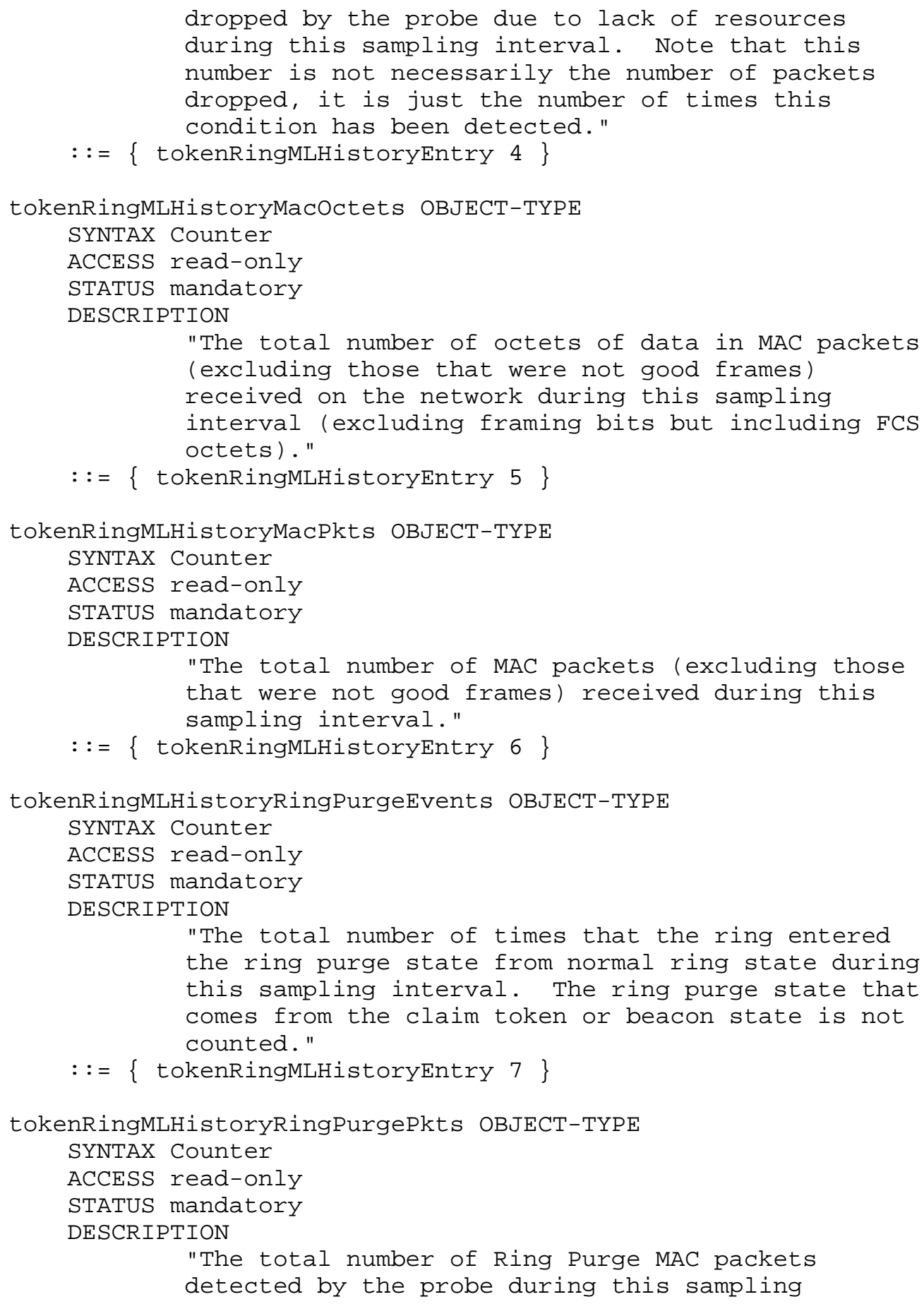




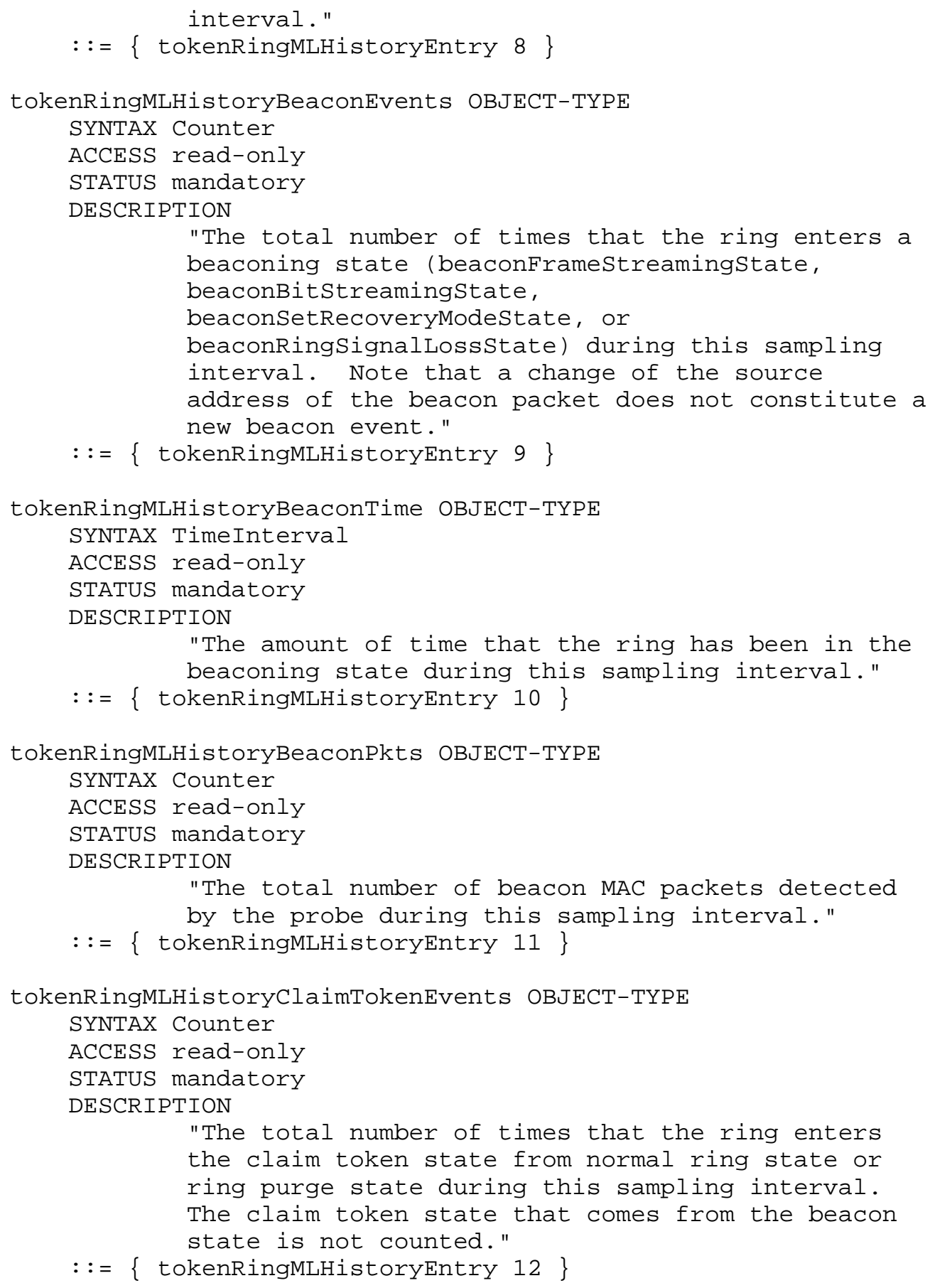









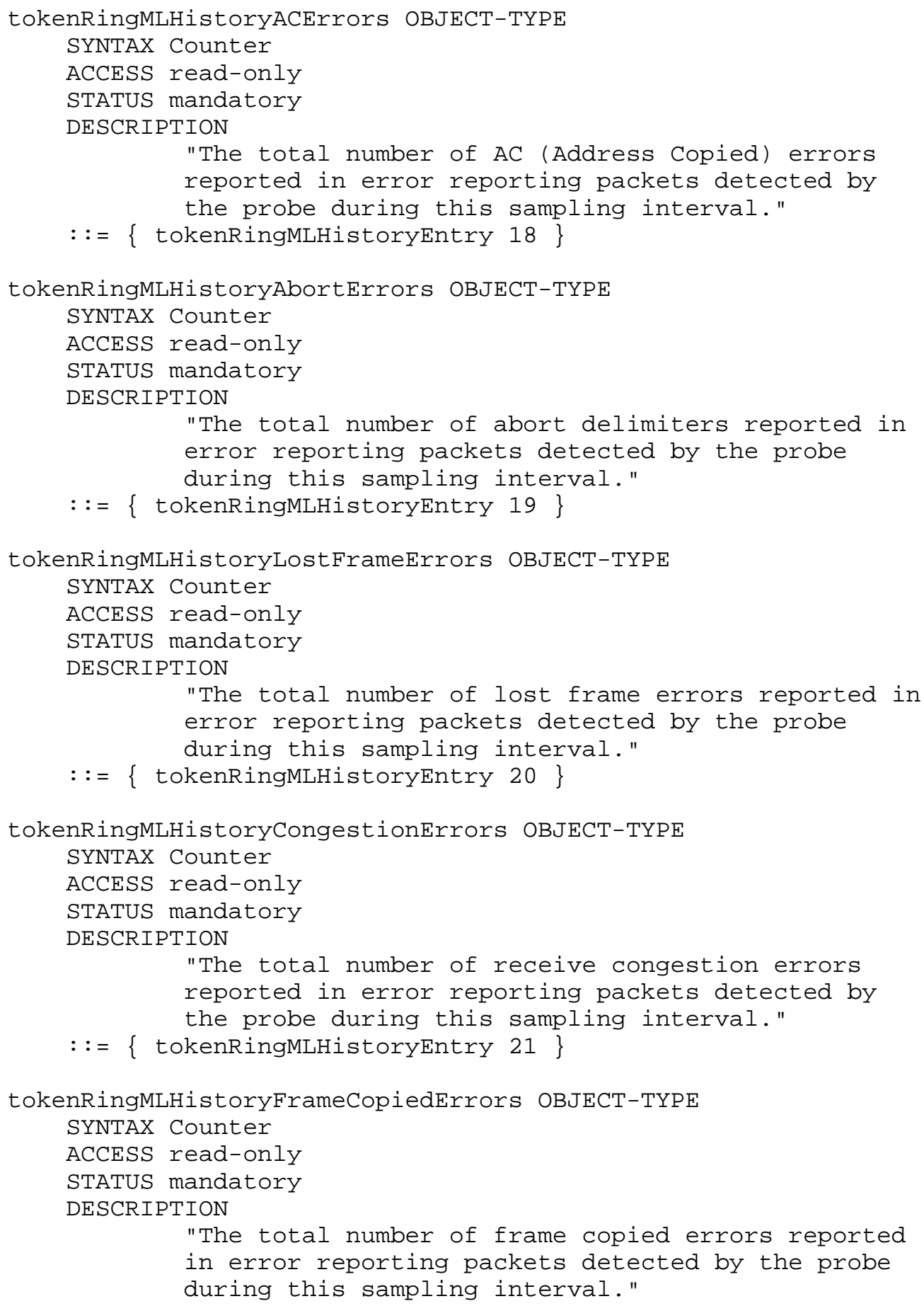




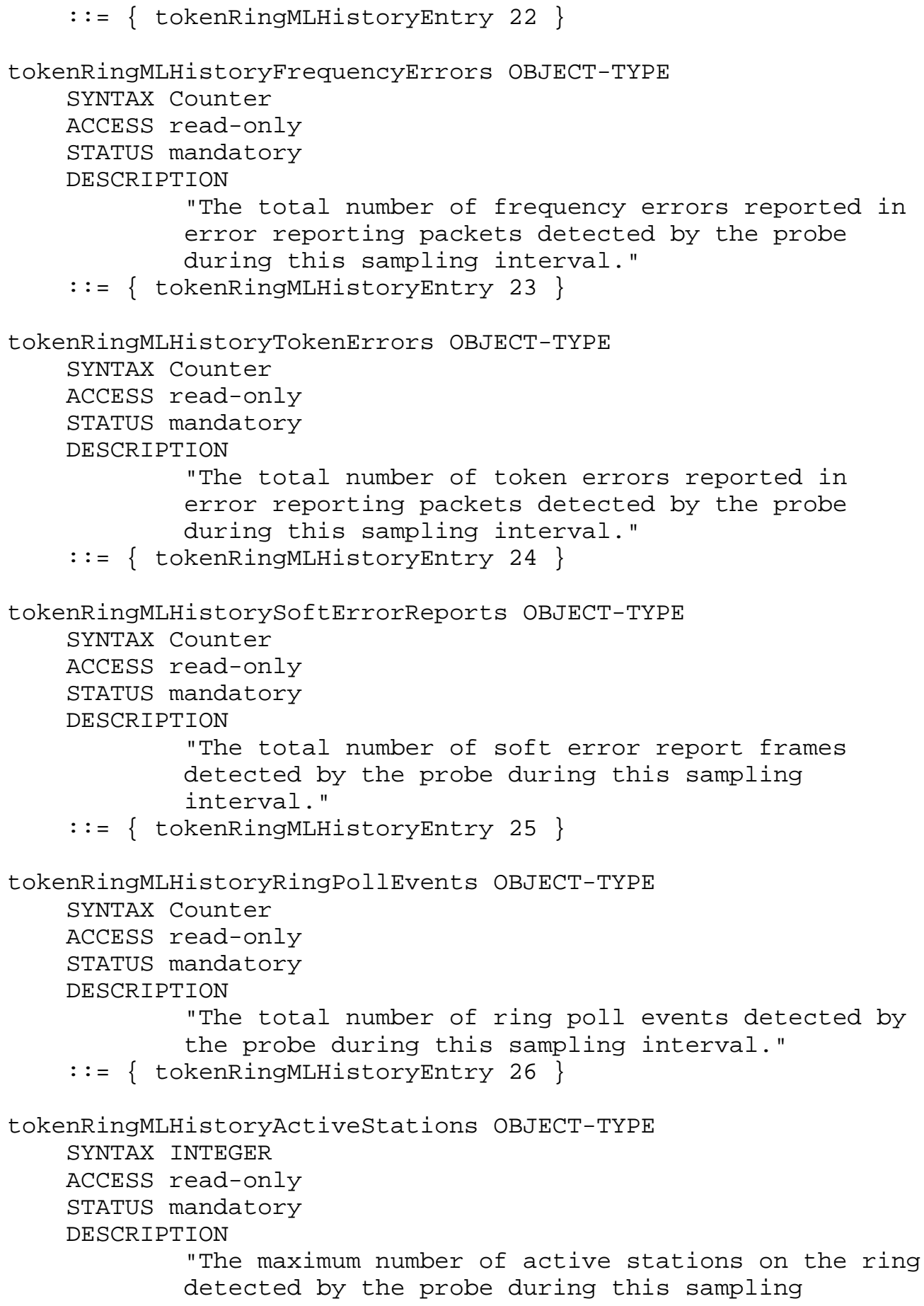




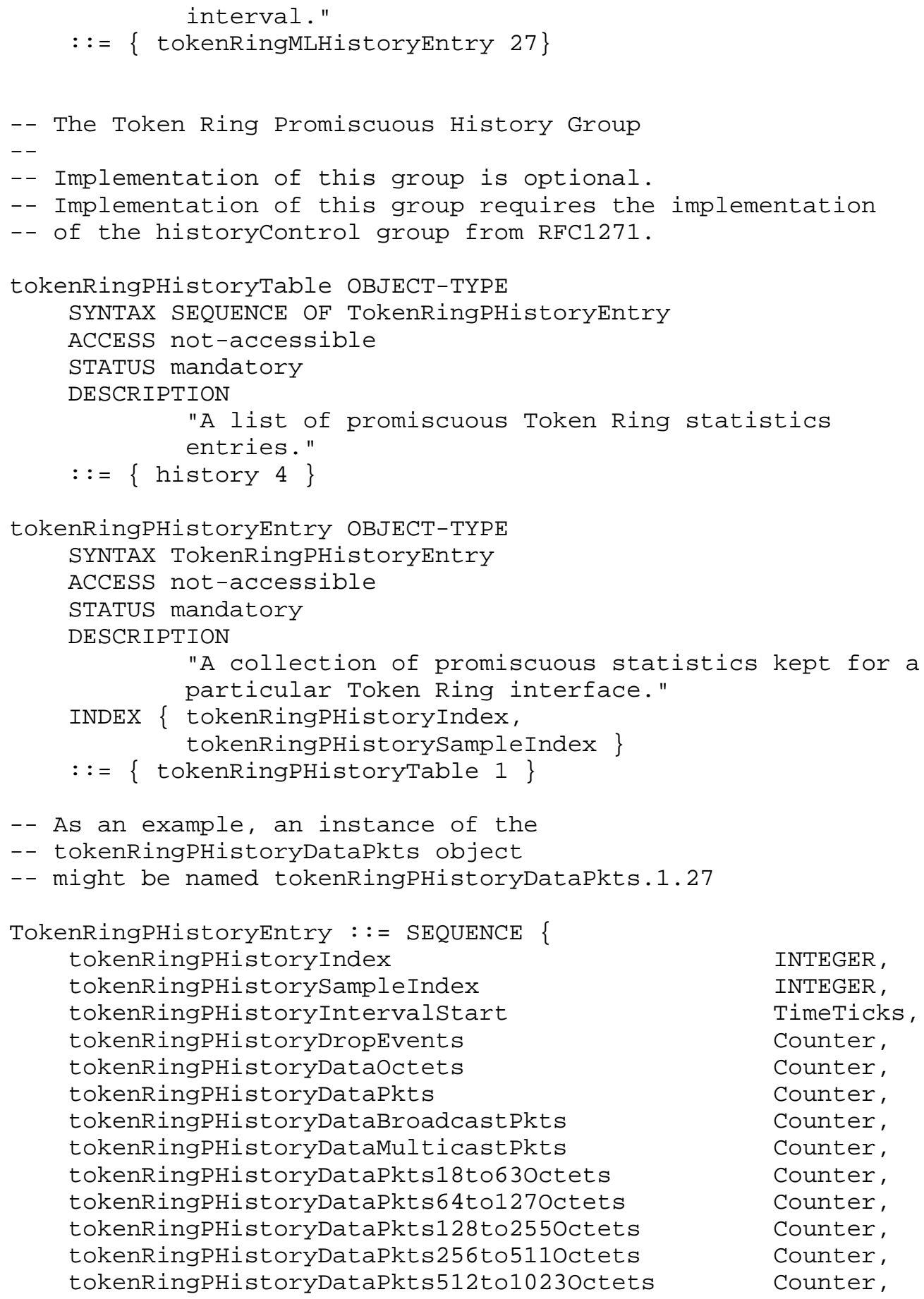

Waldbusser 


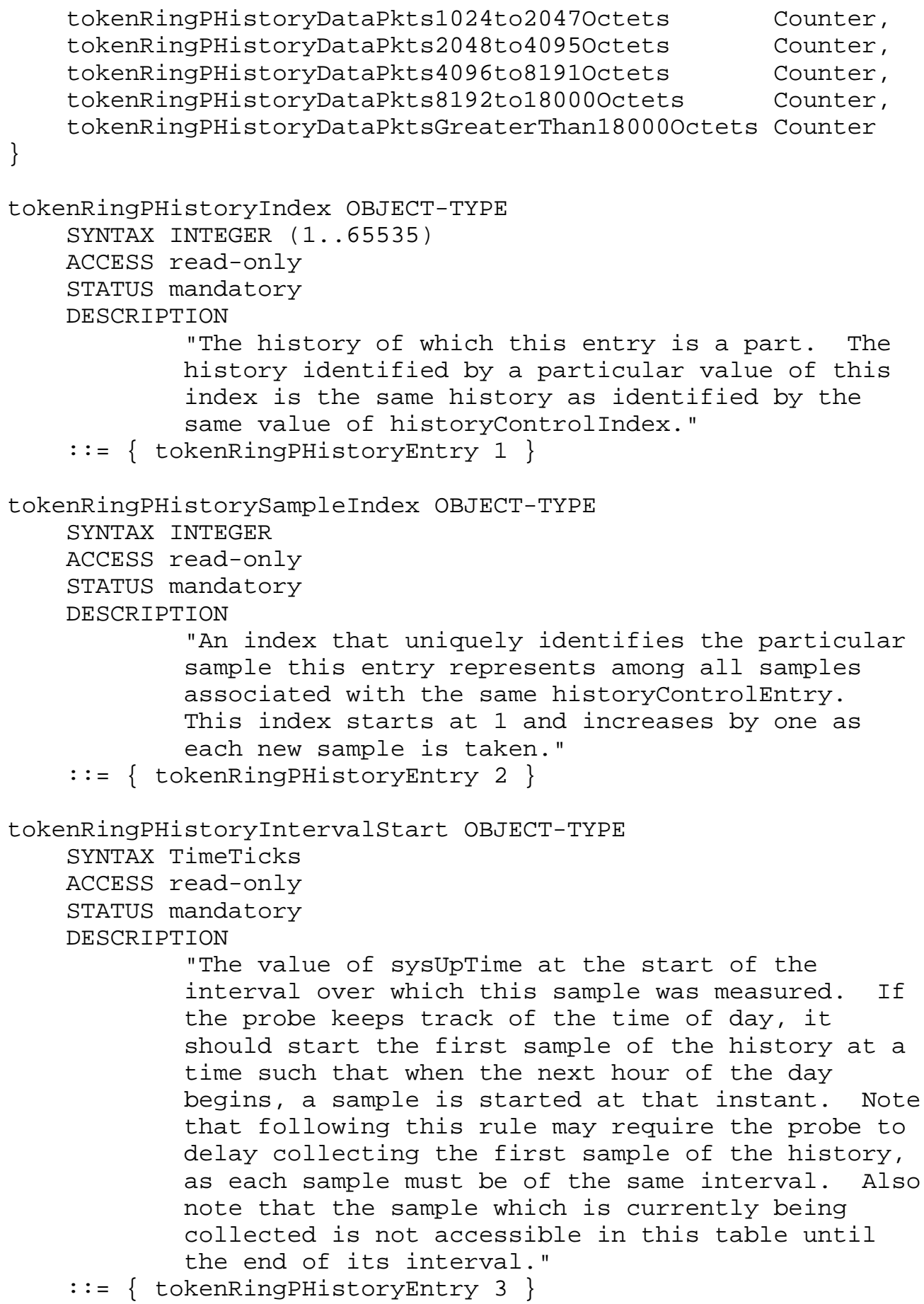

Waldbusser 


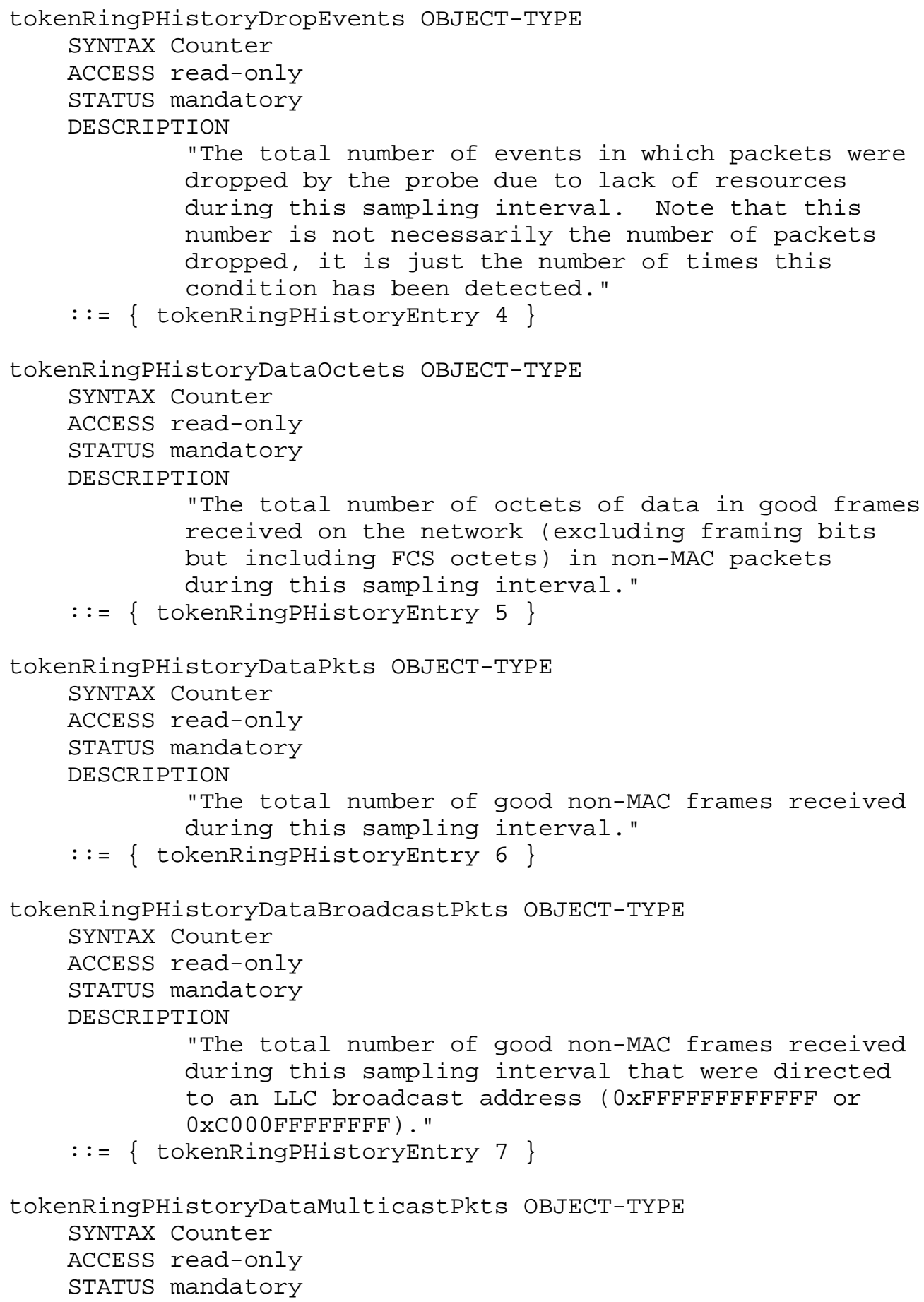




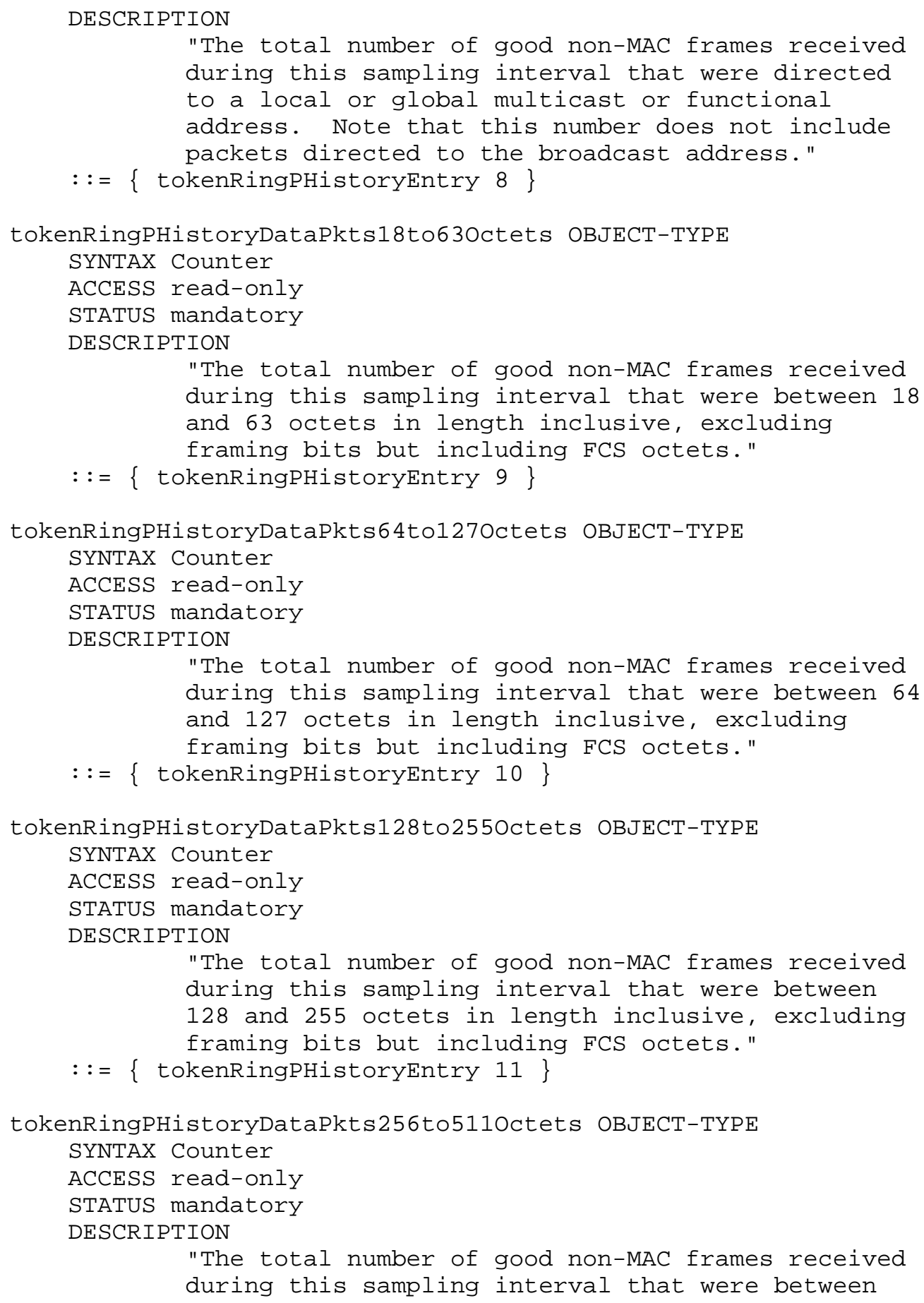




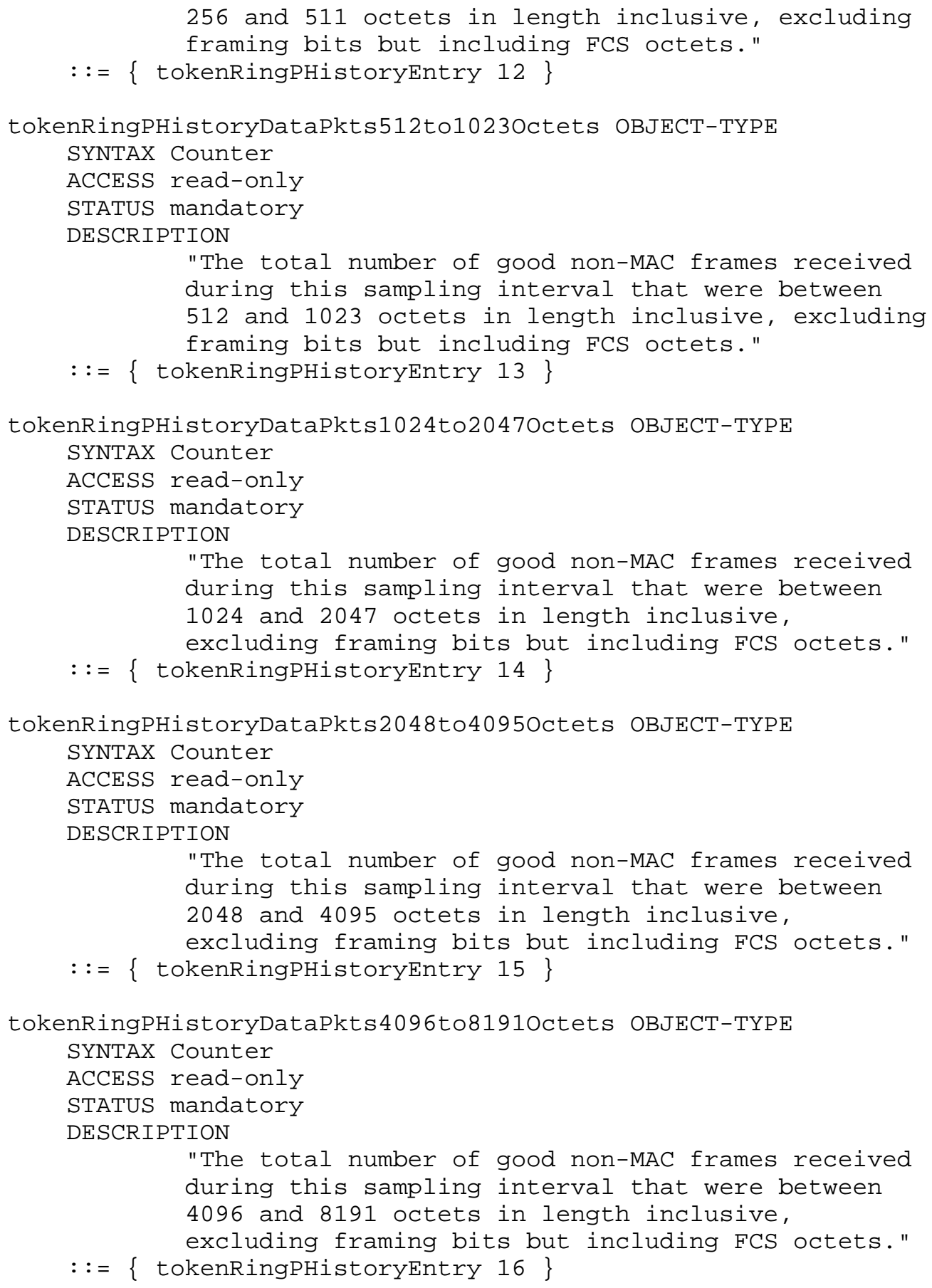




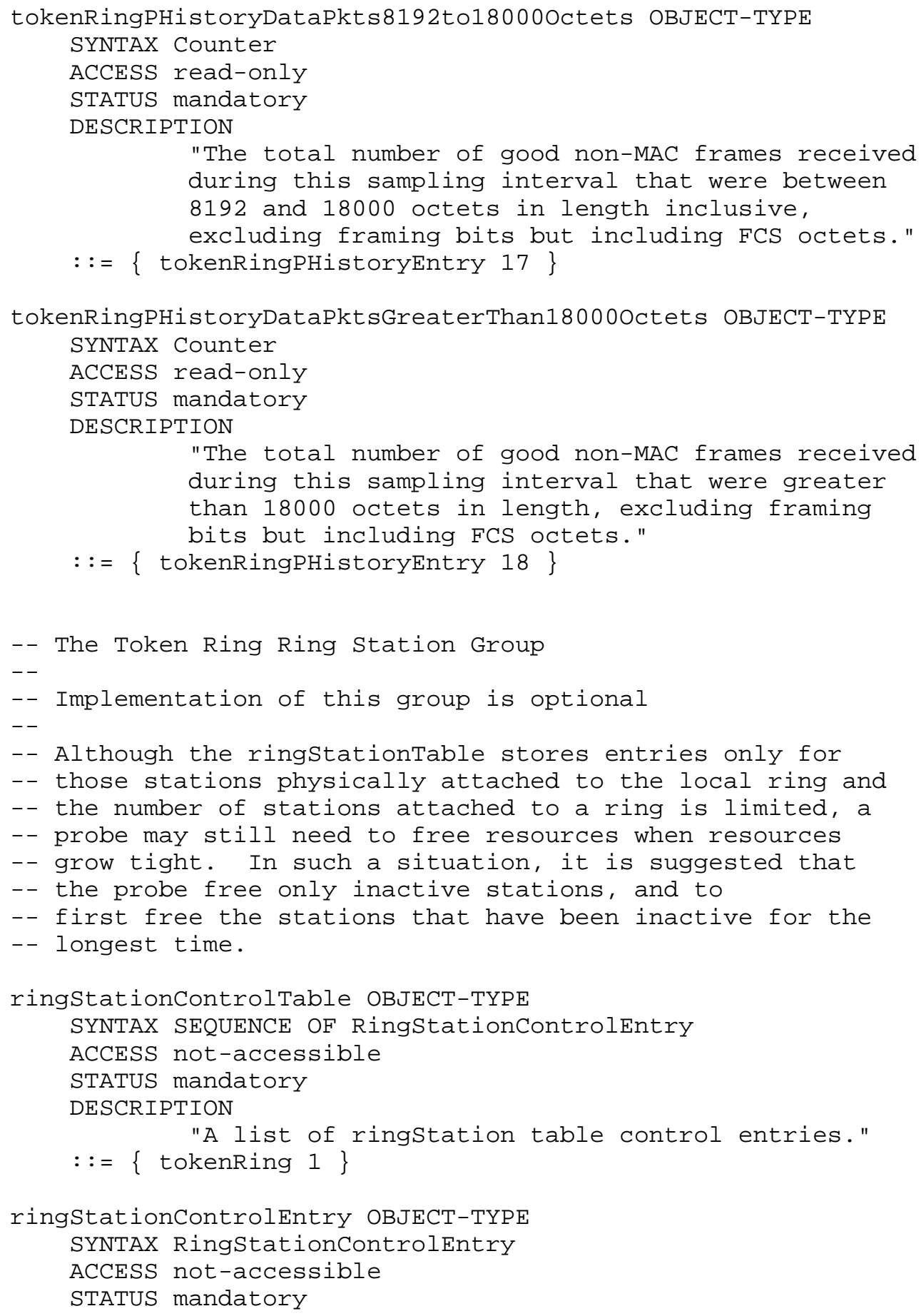




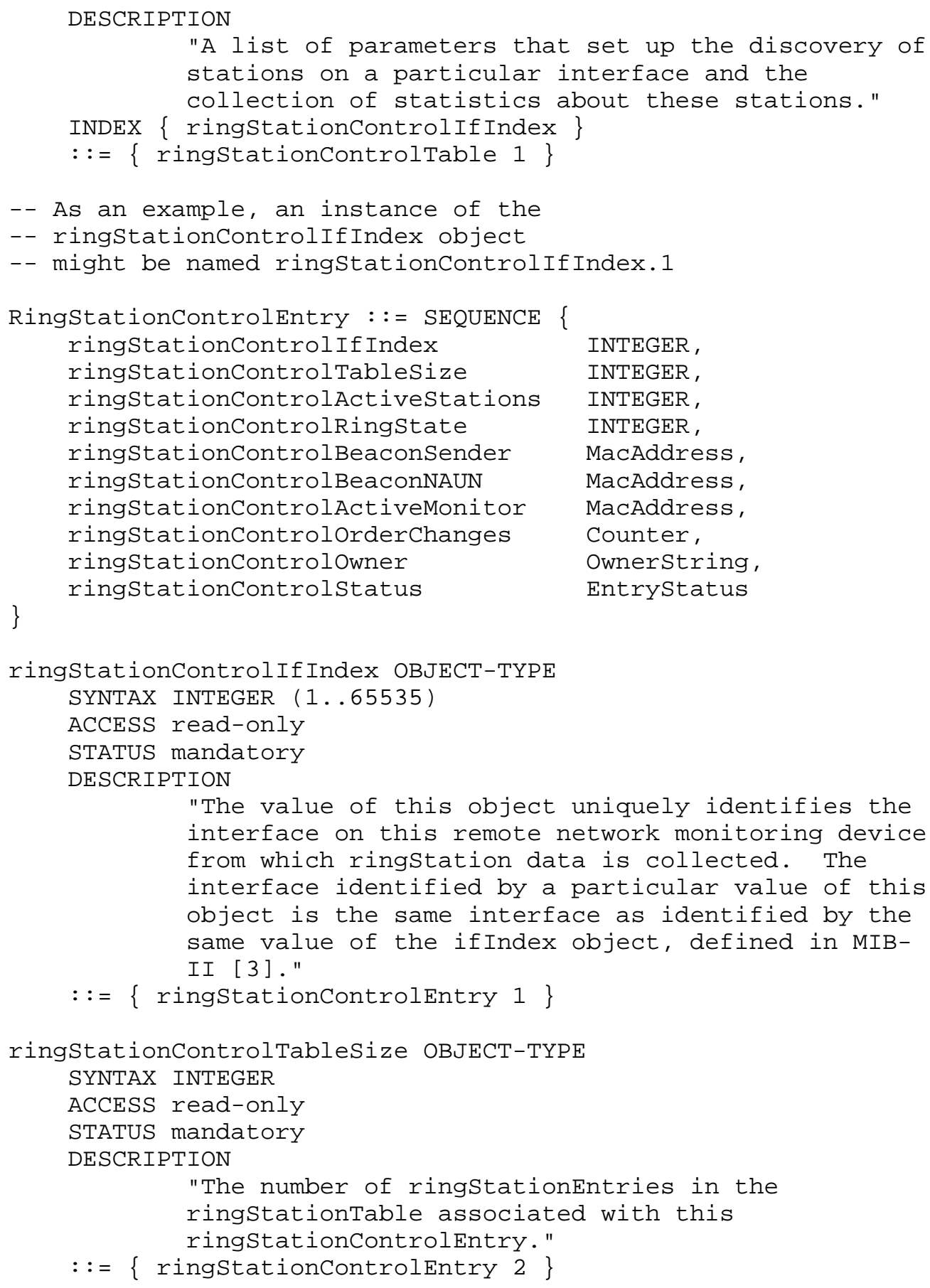




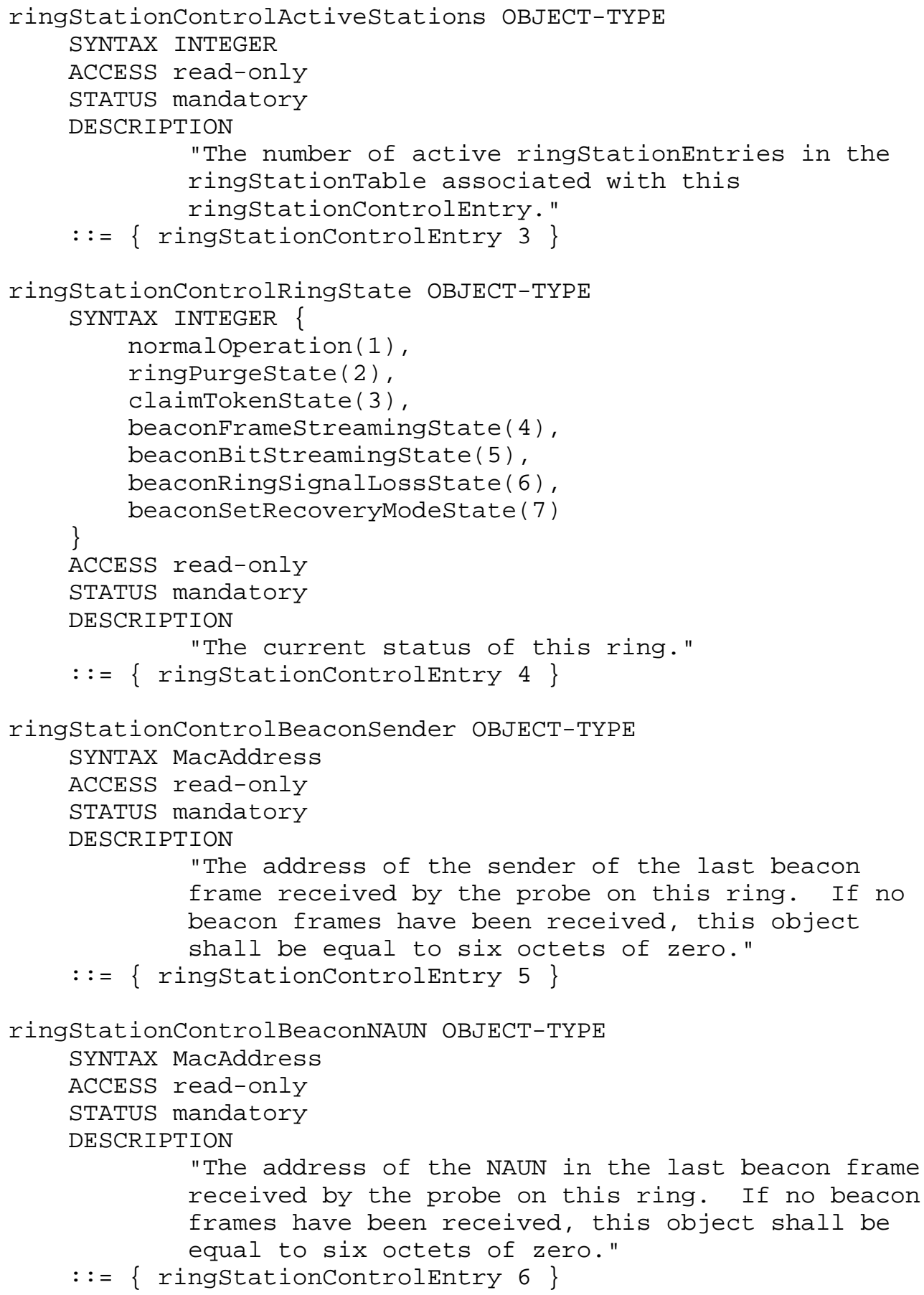




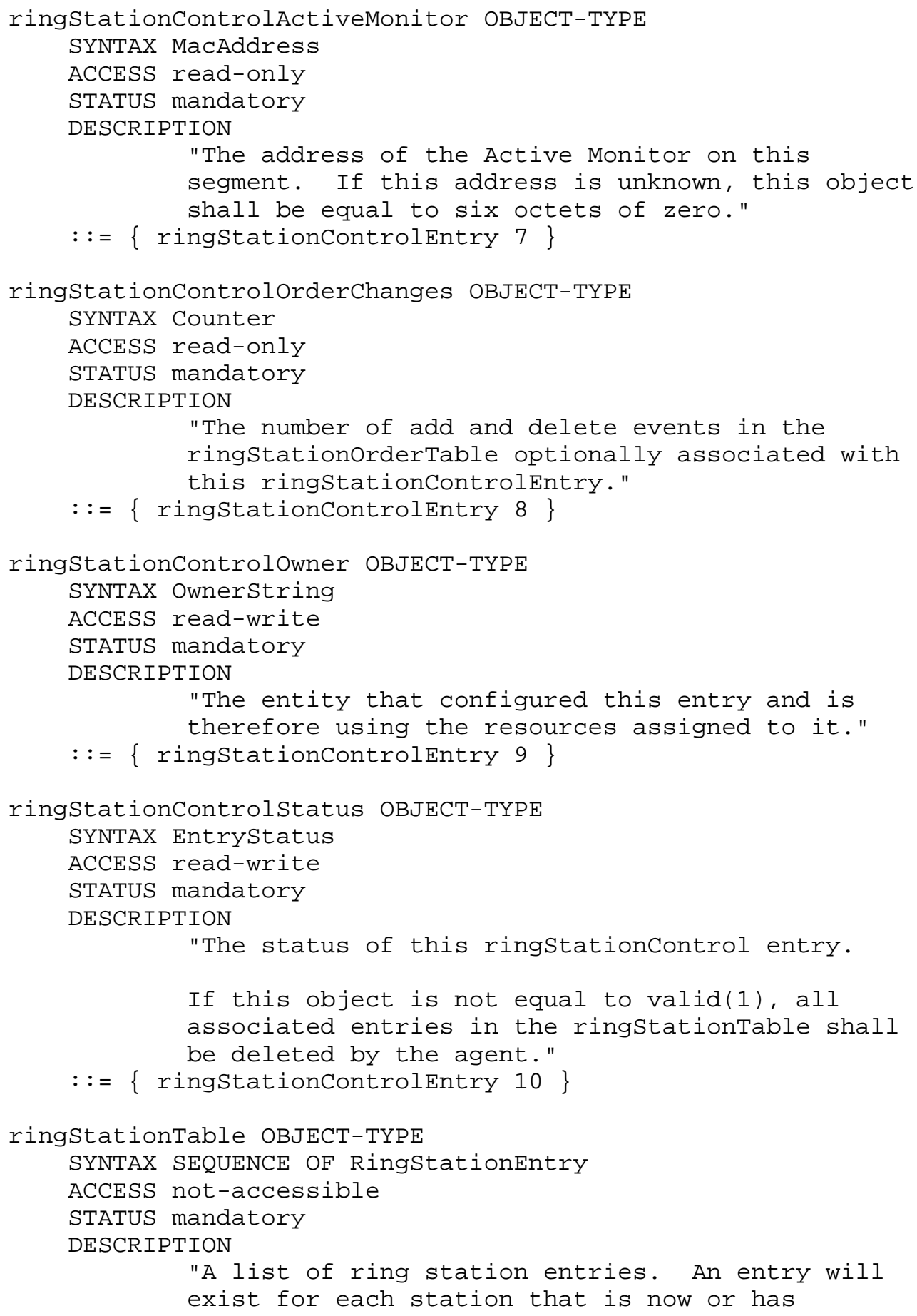




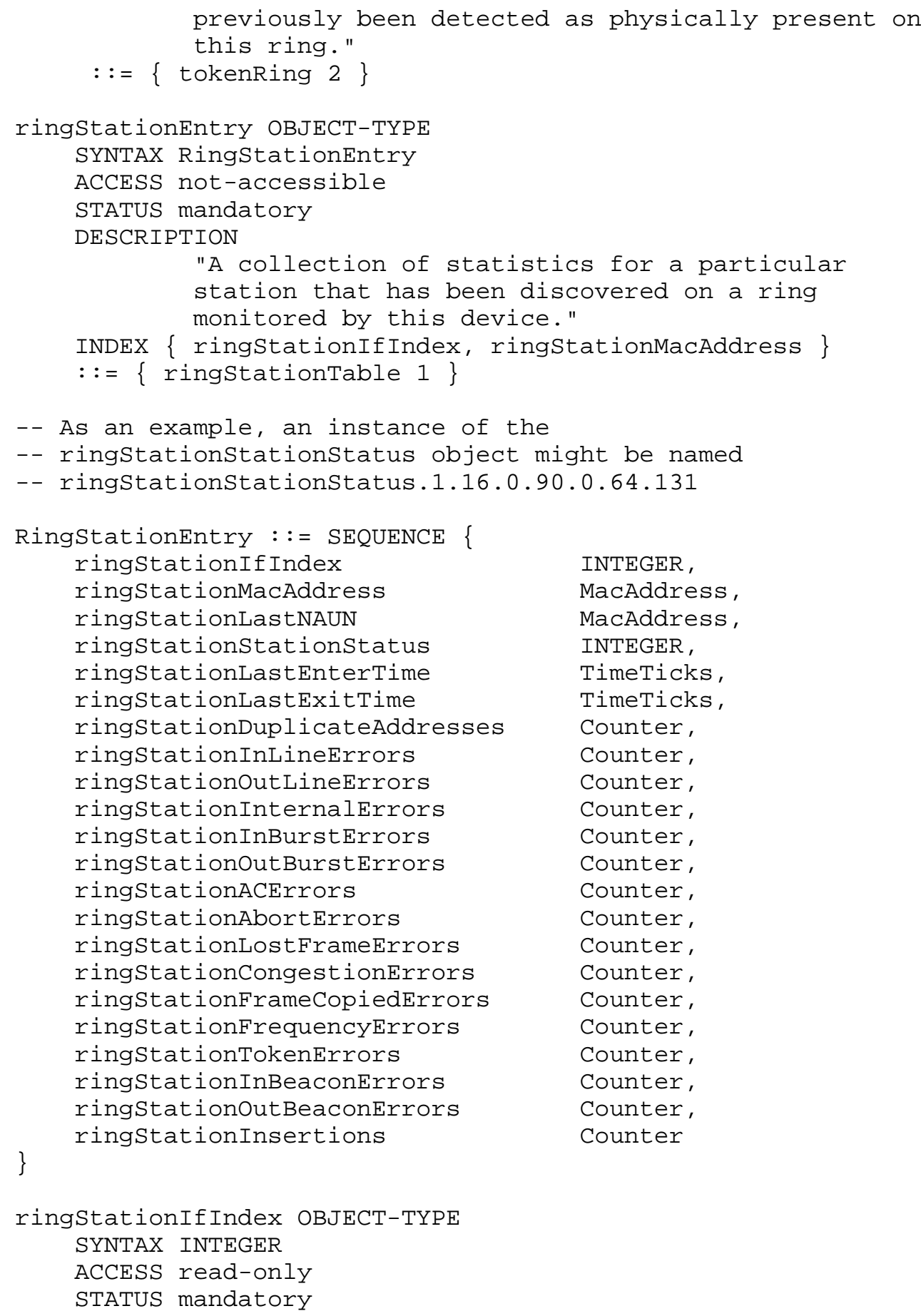

Waldbusser 


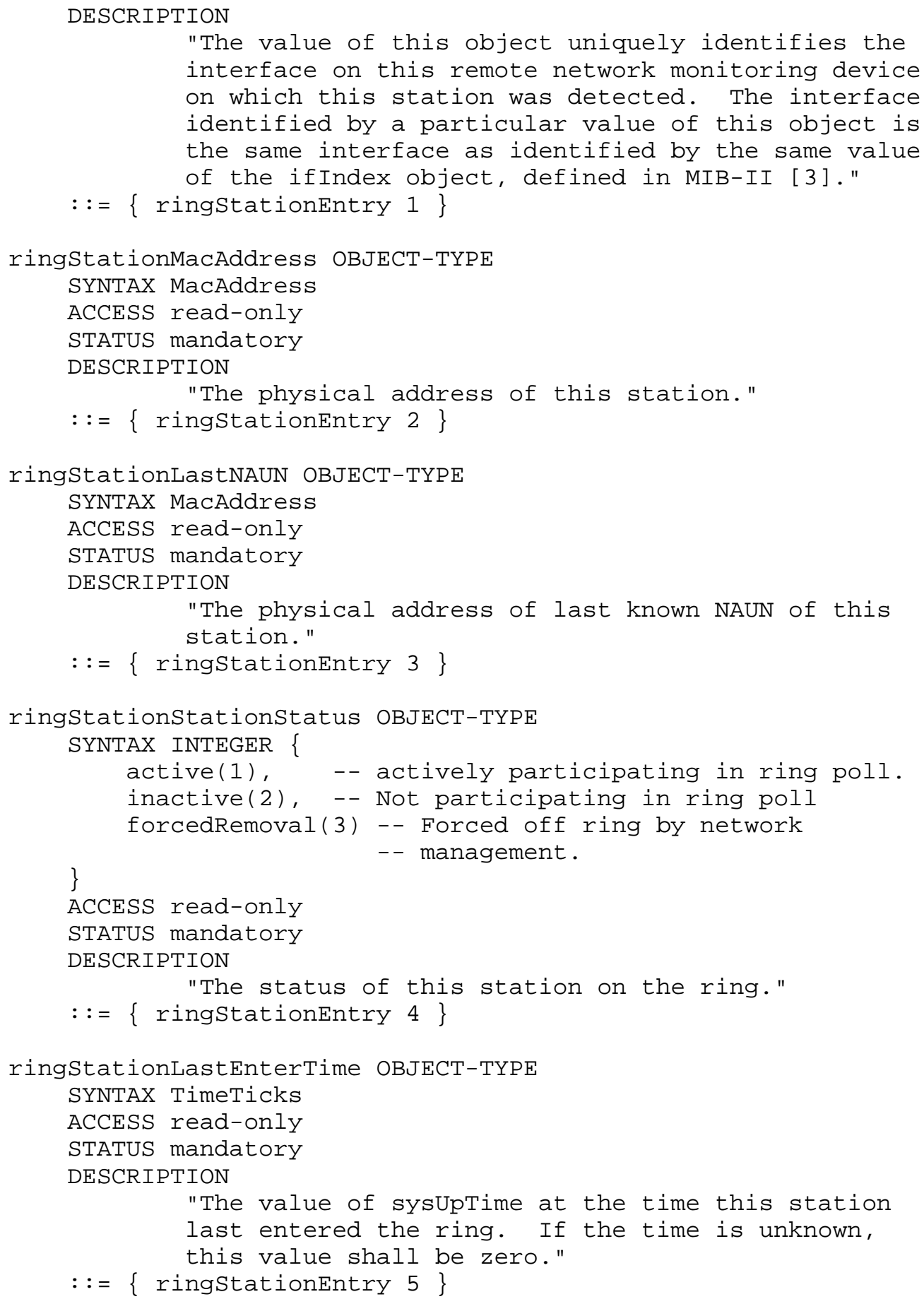




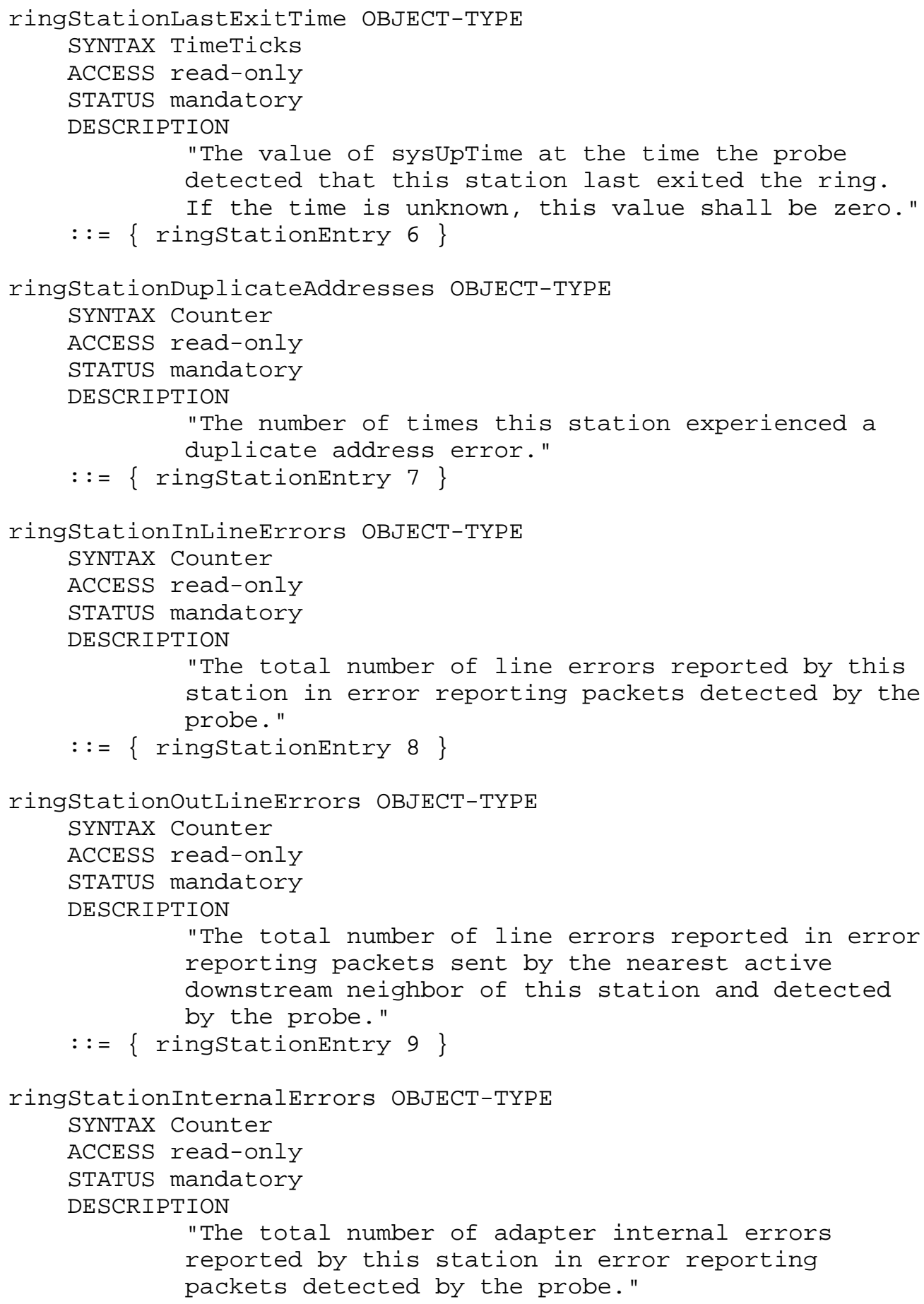




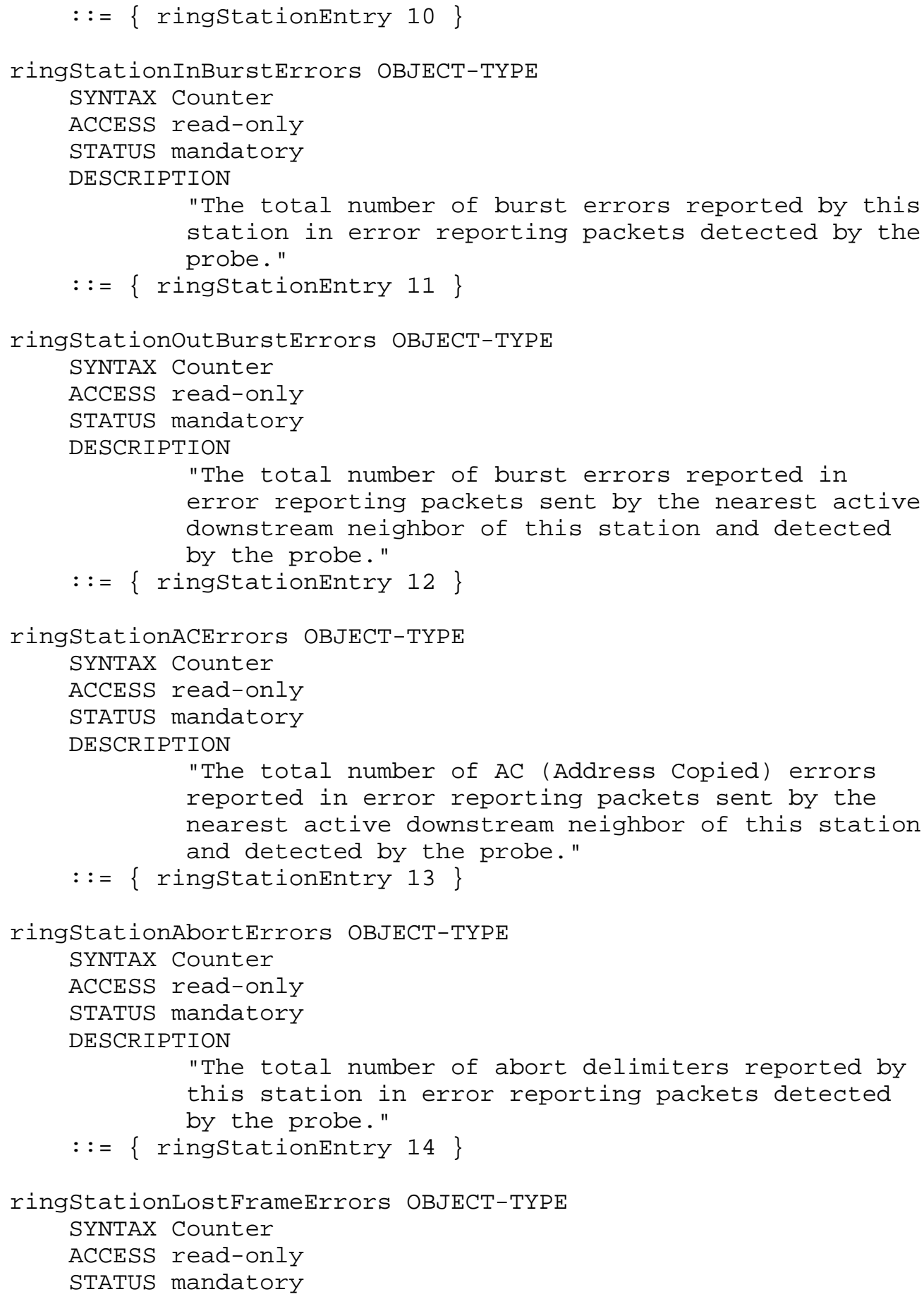




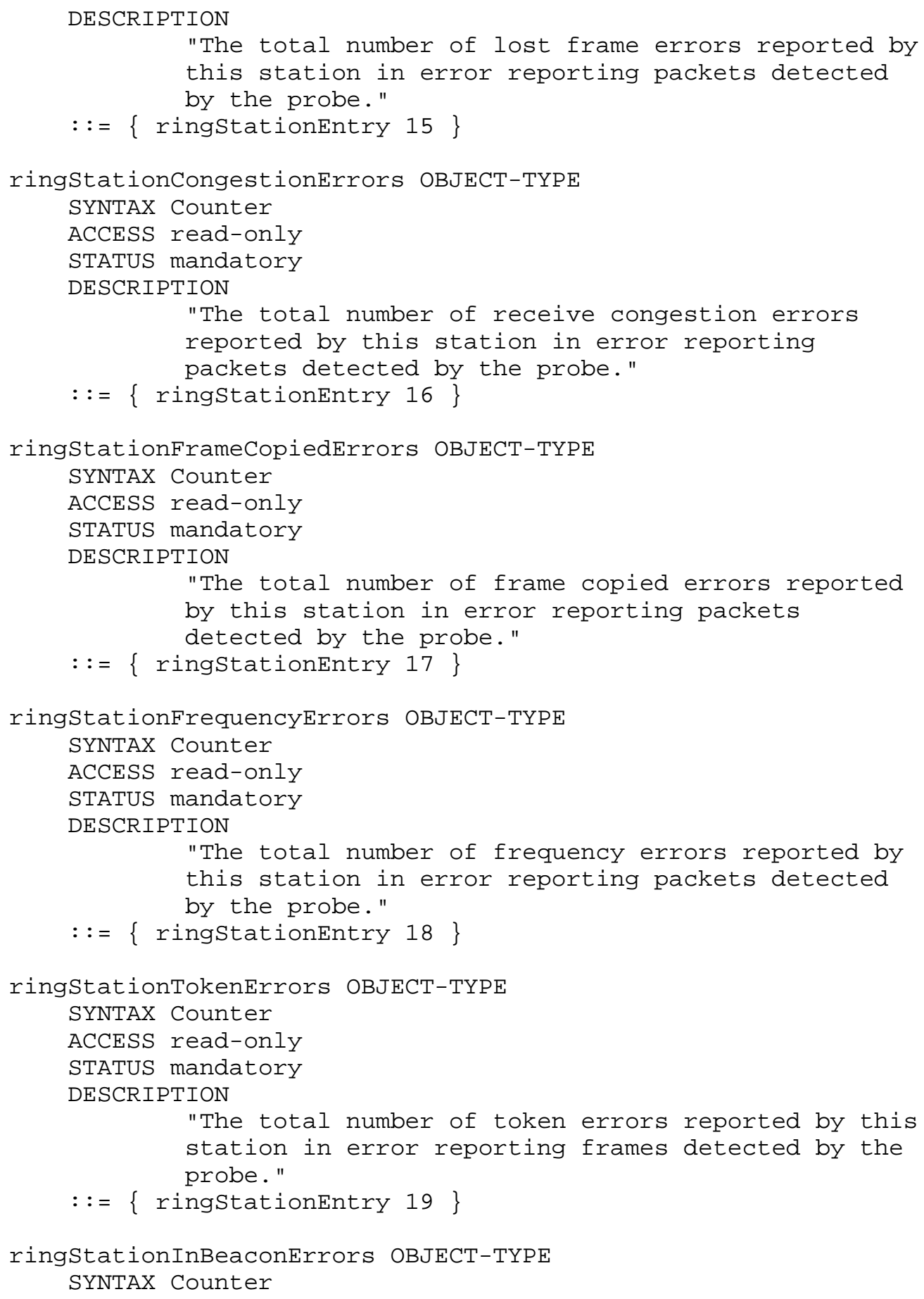






"A collection of statistics for a particular 


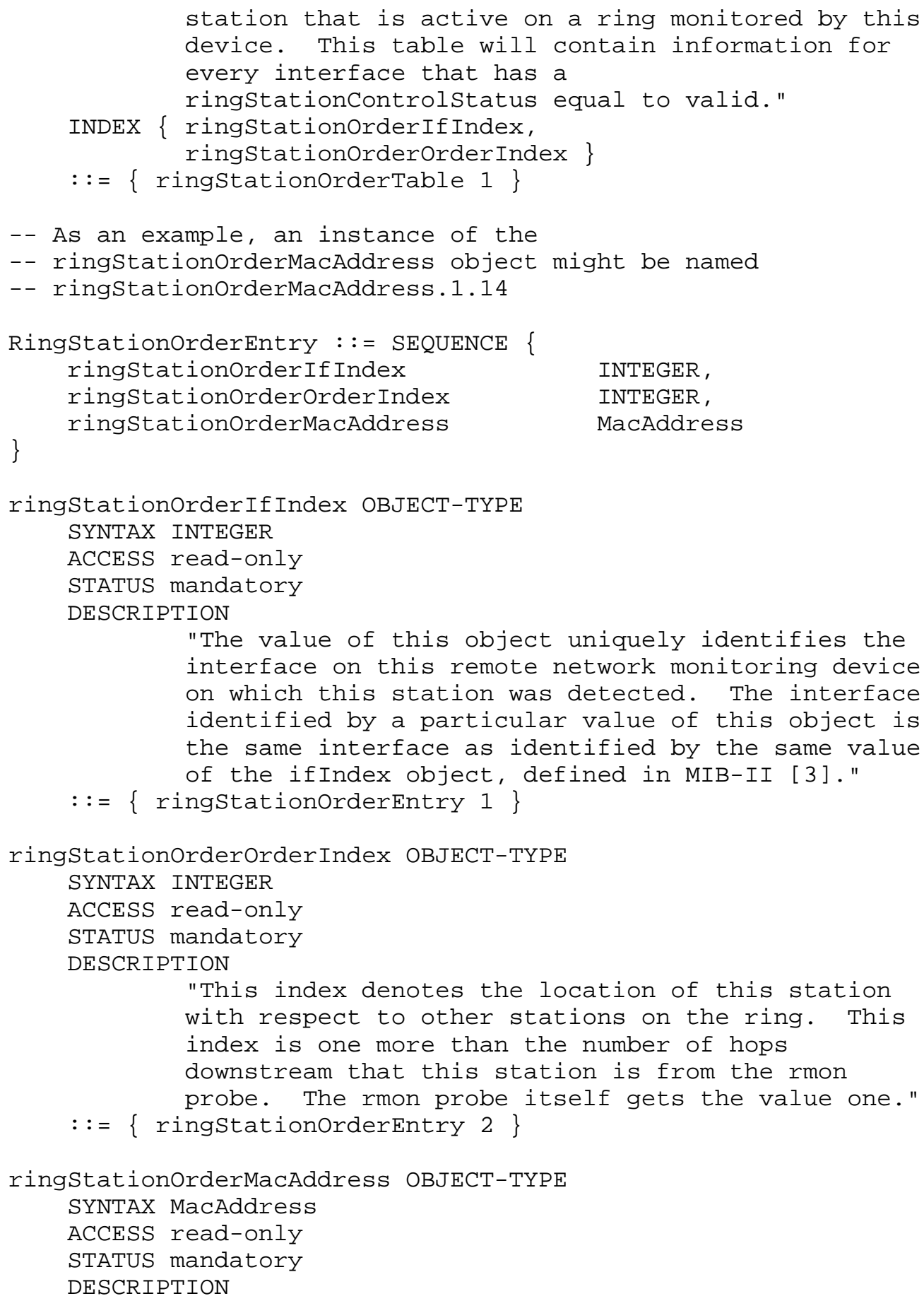









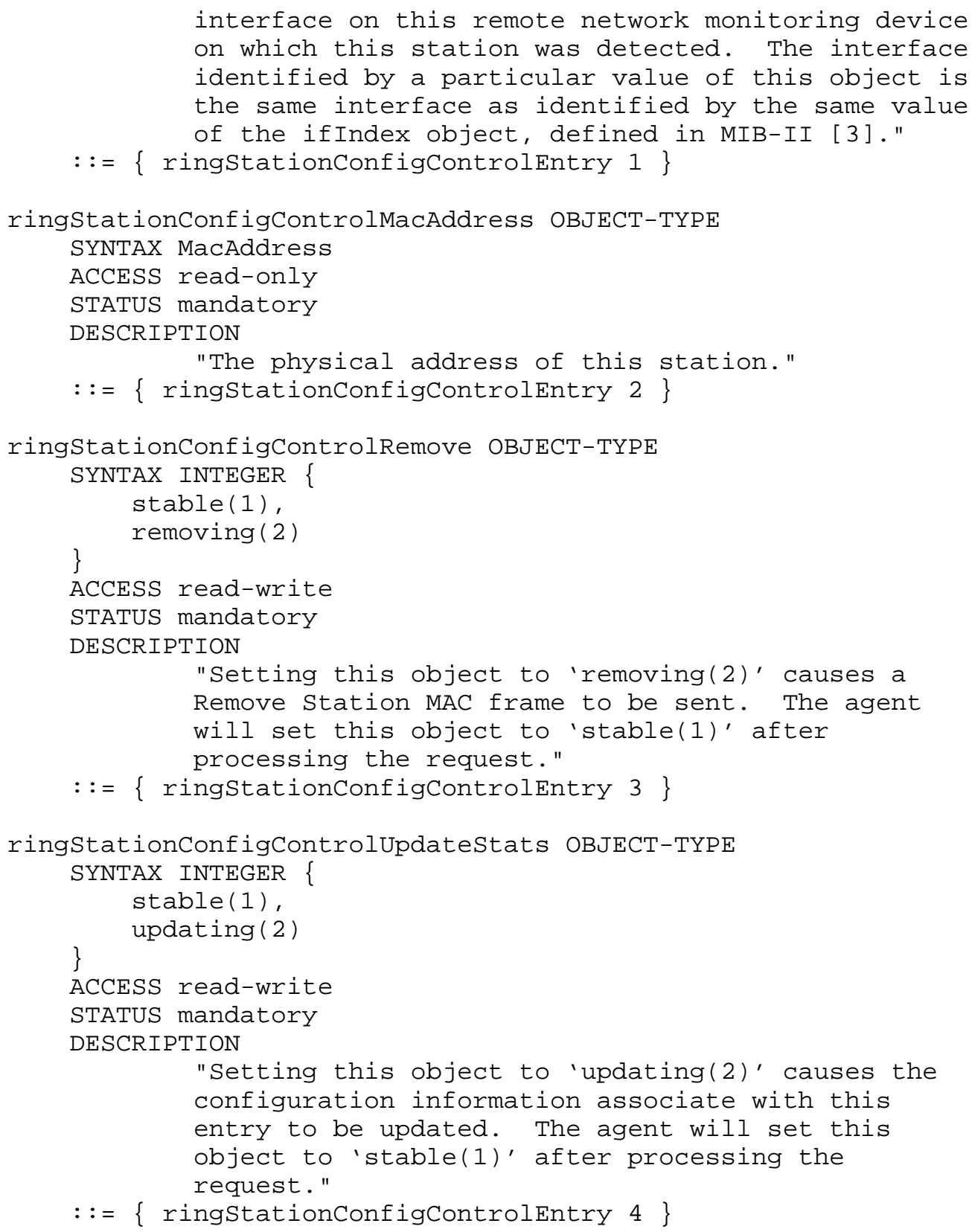


-- The ringstationconfig Table

-- Entries exist in this table after an active

-- configuration query has completed successfully for

-- a station. This query is initiated by the associated

-- ringstationConfigControlupdatestats variable.

ringstationConfigTable OBJECT-TYPE

SYNTAX SEQUENCE OF RingStationConfigEntry

ACCESS not-accessible

STATUS mandatory

DESCRIPTION

"A list of configuration entries for stations on a ring monitored by this probe."

$::=\{$ tokenRing 5$\}$

ringStationConfigEntry OBJECT-TYPE

SYNTAX RingStationConfigEntry

ACCESS not-accessible

STATUS mandatory

DESCRIPTION

"A collection of statistics for a particular

station that has been discovered on a ring monitored by this probe."

INDEX \{ ringstationConfigIfIndex, ringStationConfigMacAddress \}

$::=\{$ ringstationConfigTable 1$\}$

-- As an example, an instance of the

-- ringStationConfiglocation object might be named

-- ringStationConfigLocation.1.16.0.90.0.64.131

RingStationConfigEntry ::= SEQUENCE \{ ringStationConfigIfIndex ringstationConfigMacAddress

ringstationConfigupdateTime

INTEGER, ringstationConfiglocation ringstationConfigMicrocode ringStationConfigGroupAddress ringstationConfigfunctionalAddress MacAddress, TimeTicks, OCTET STRING, OCTET STRING, OCTET STRING, \} OCTET STRING

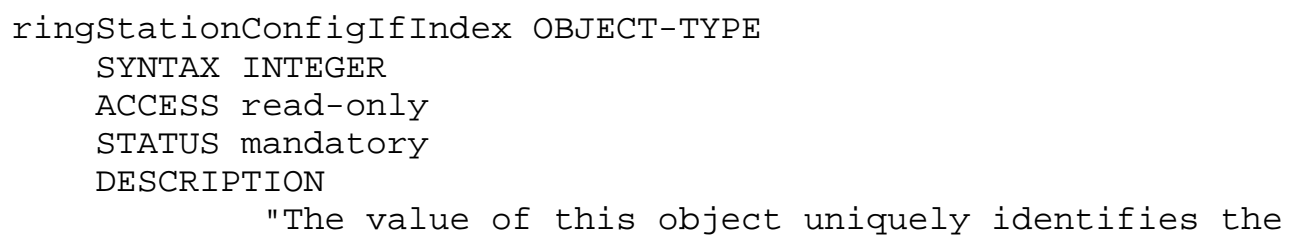

"The value of this object uniquely identifies the 


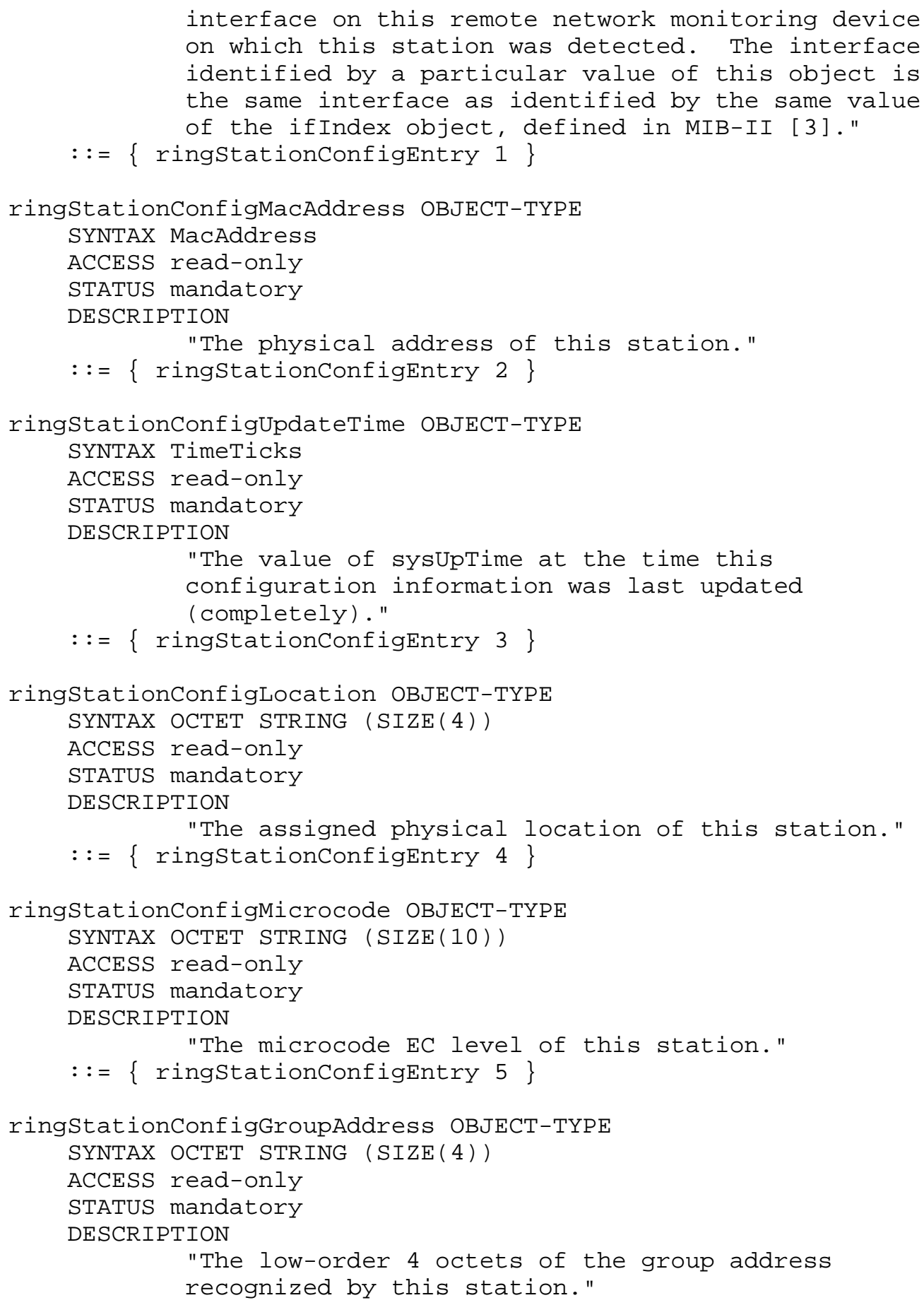




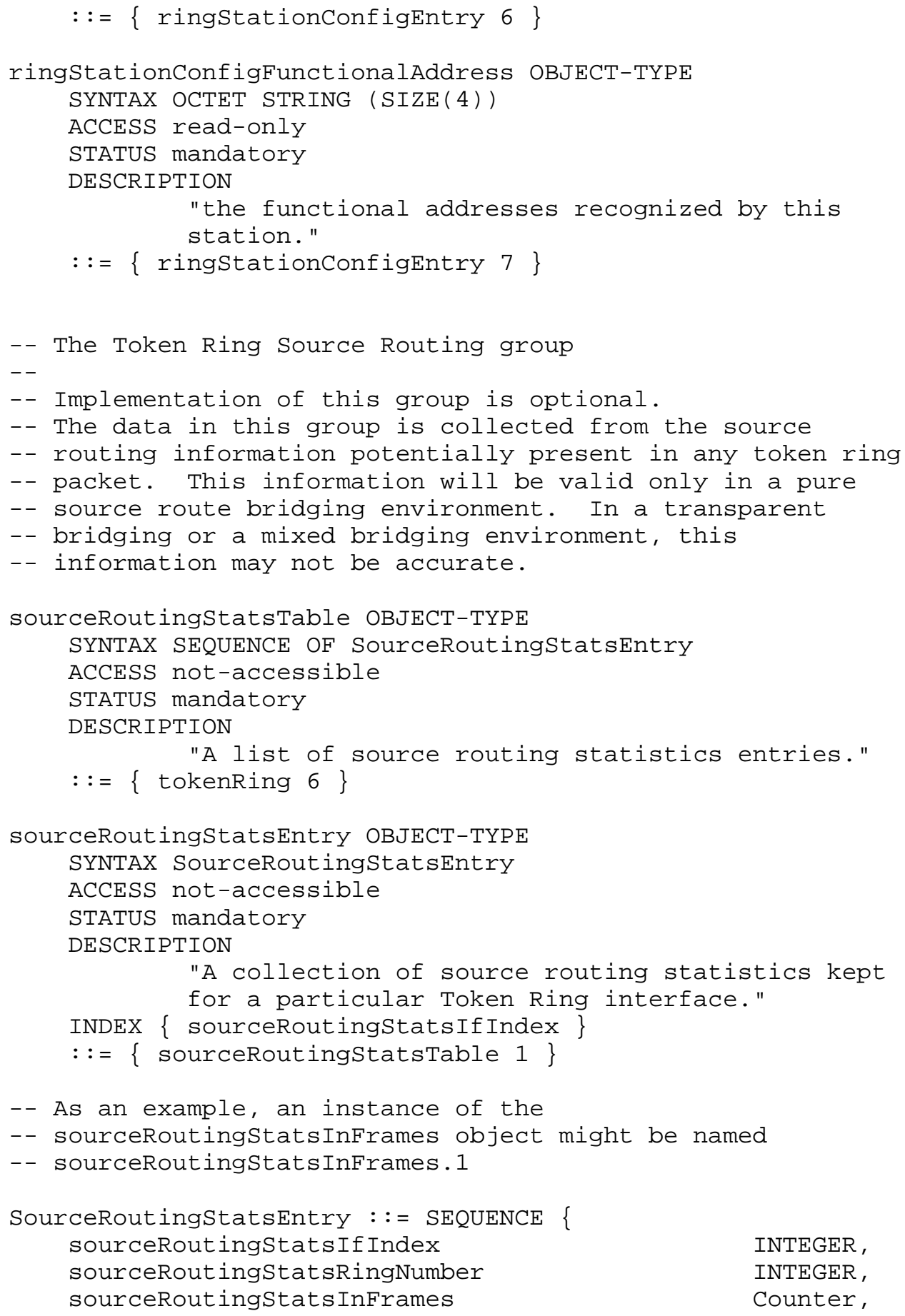




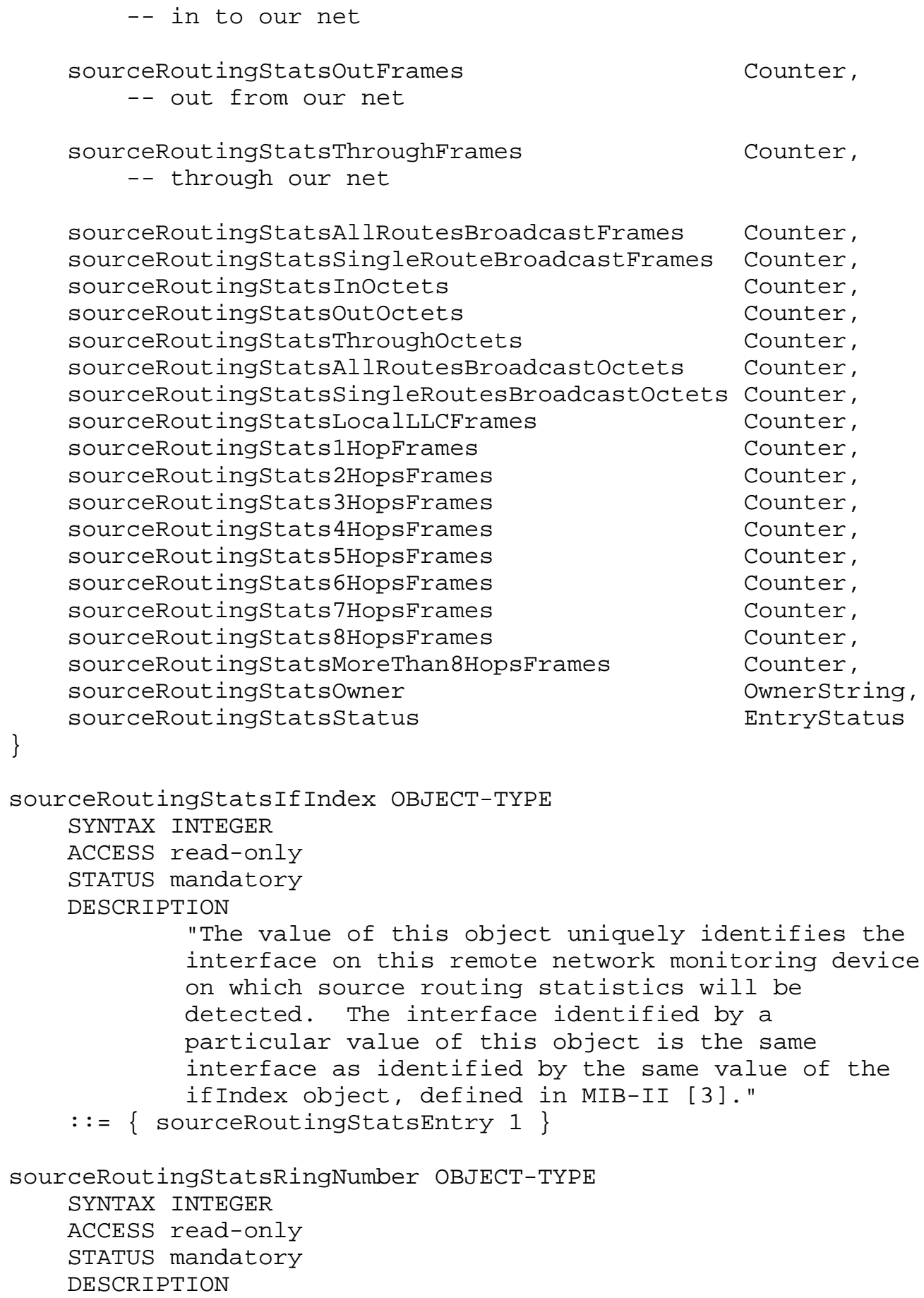




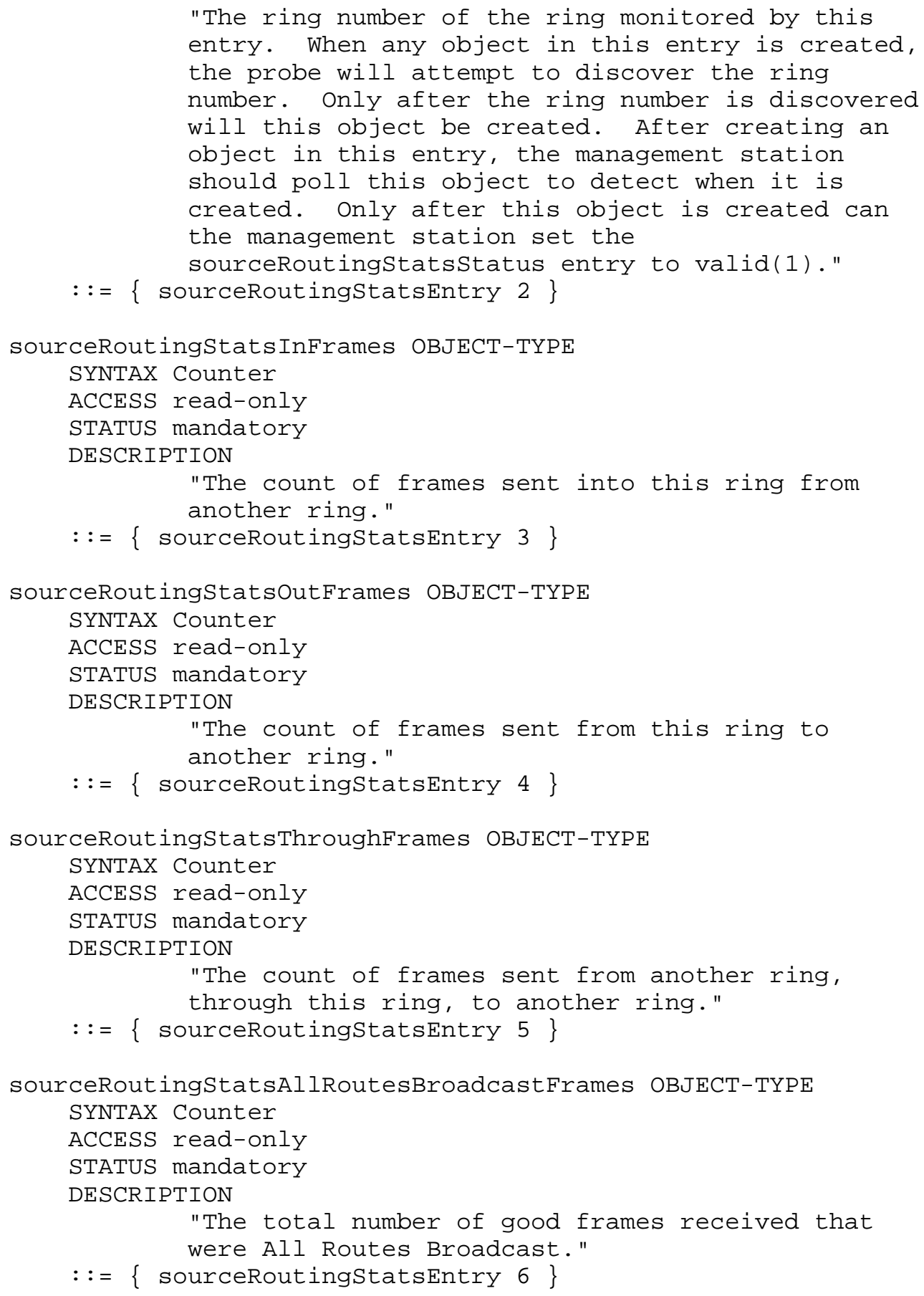




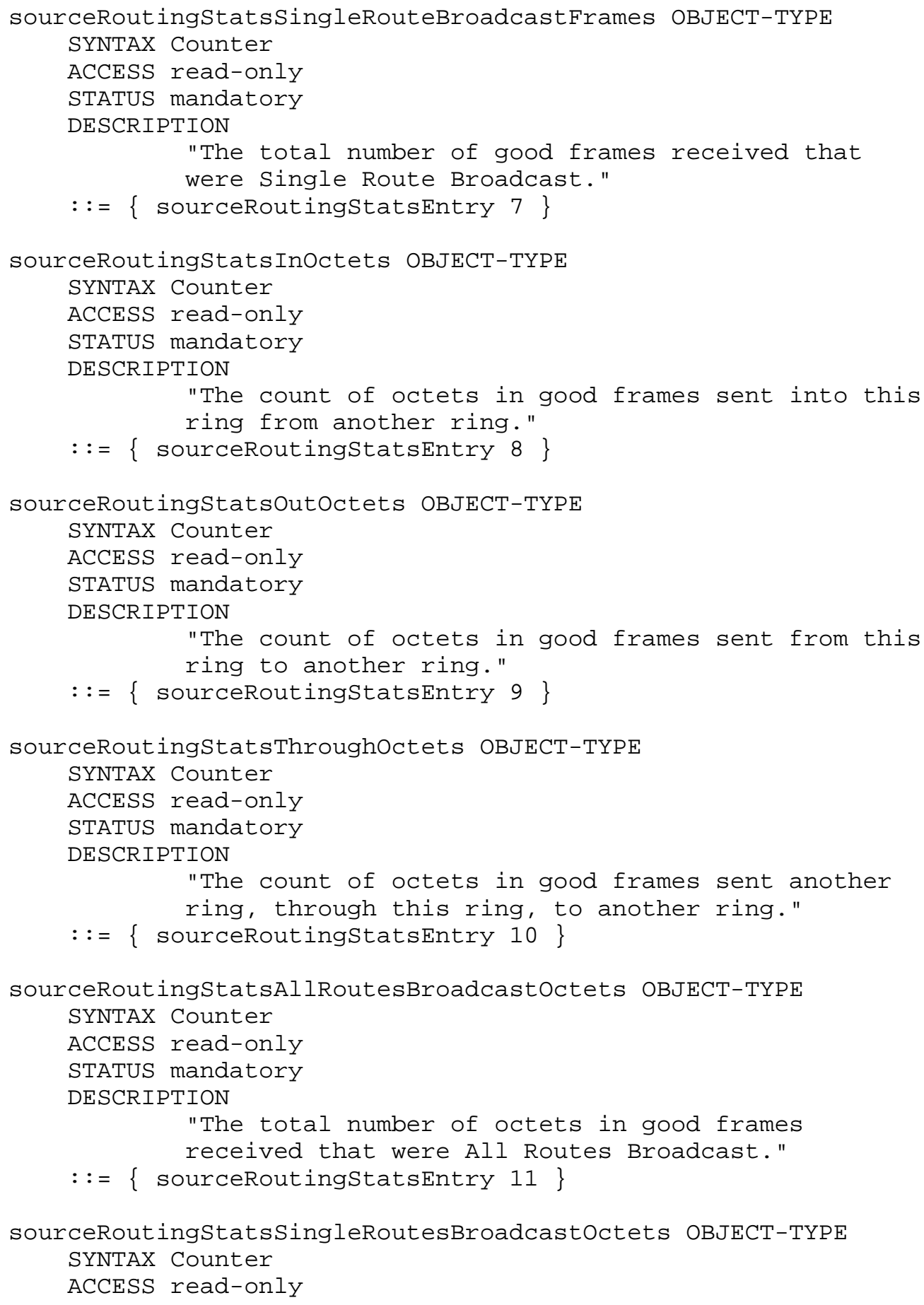




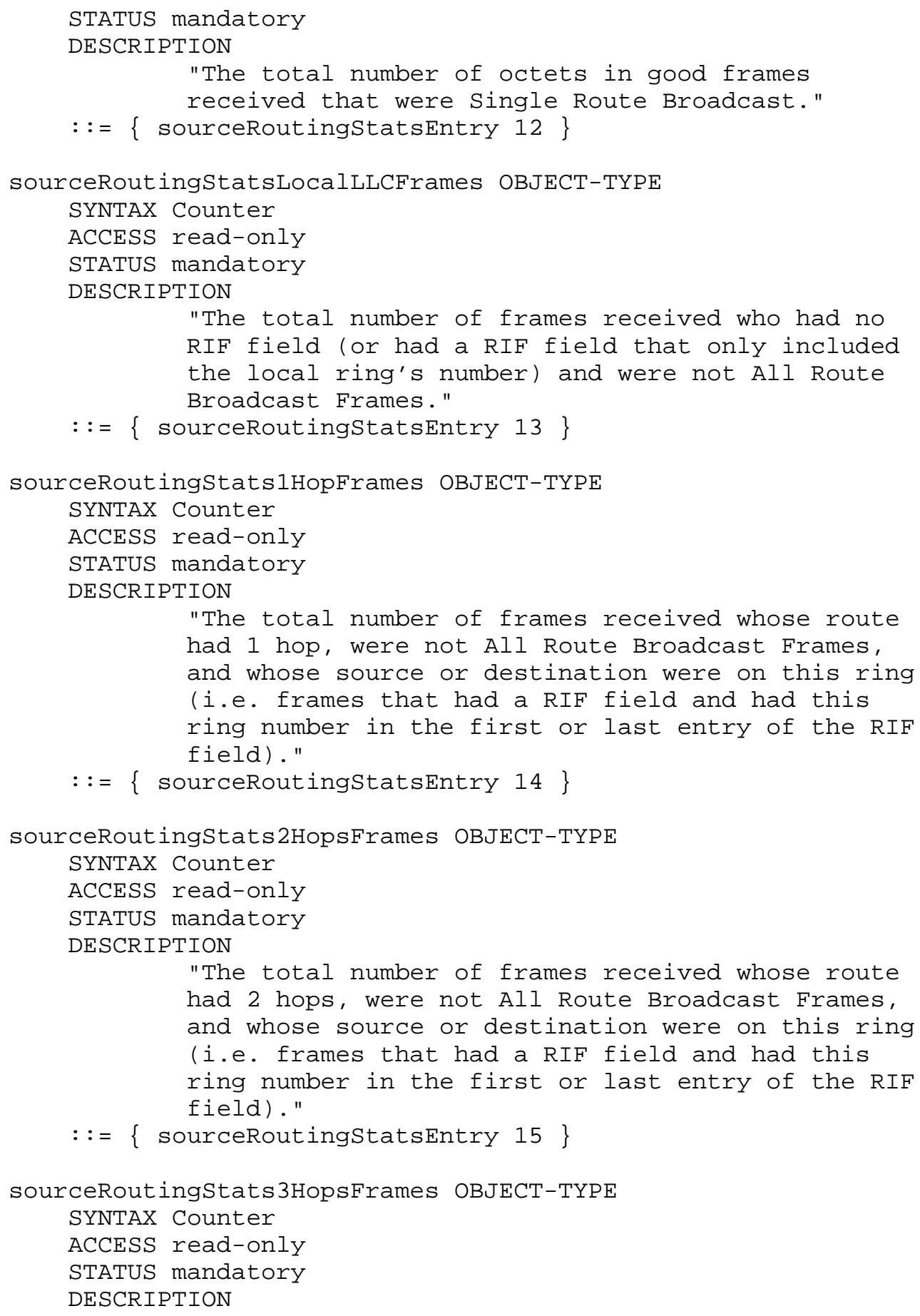




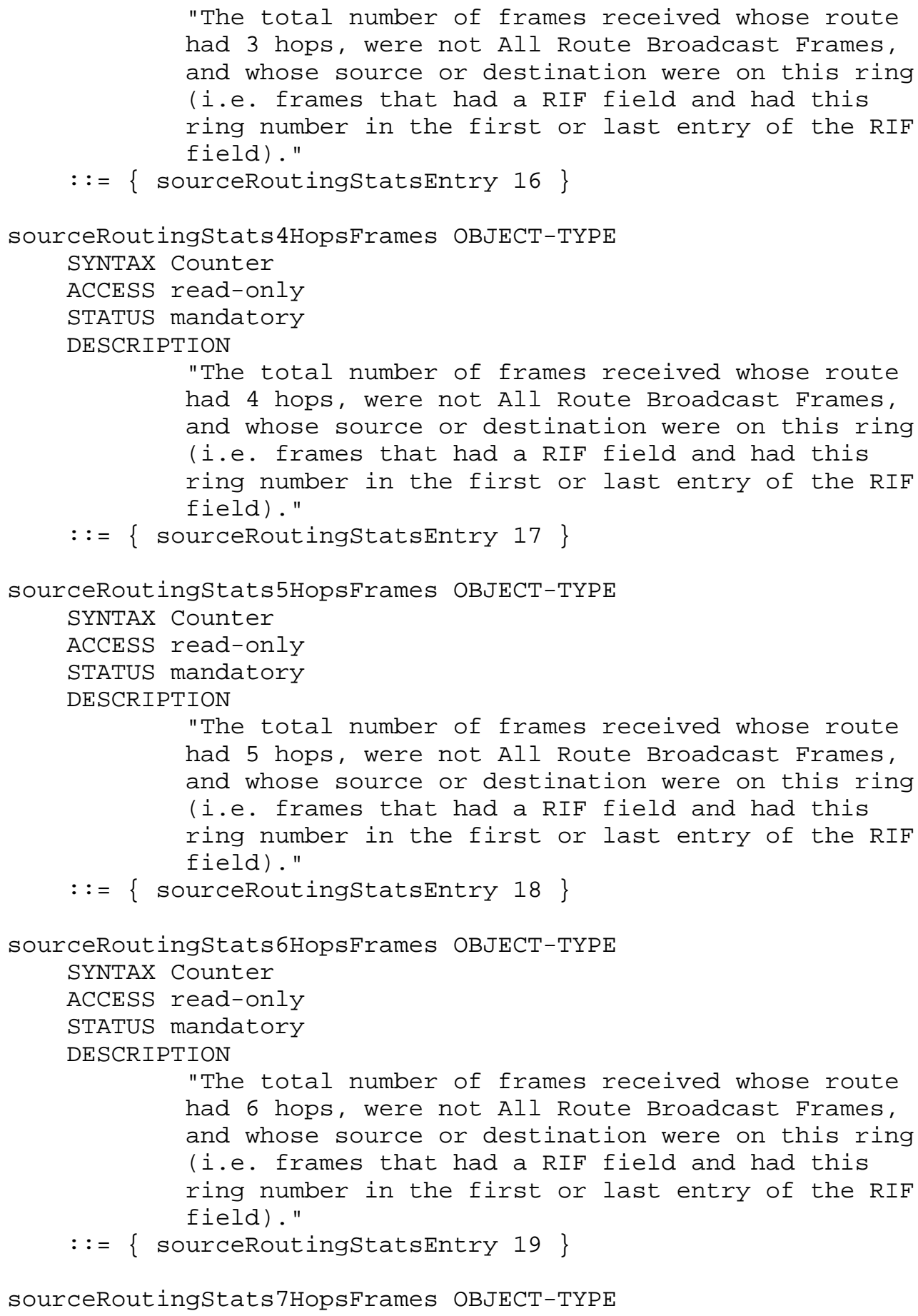




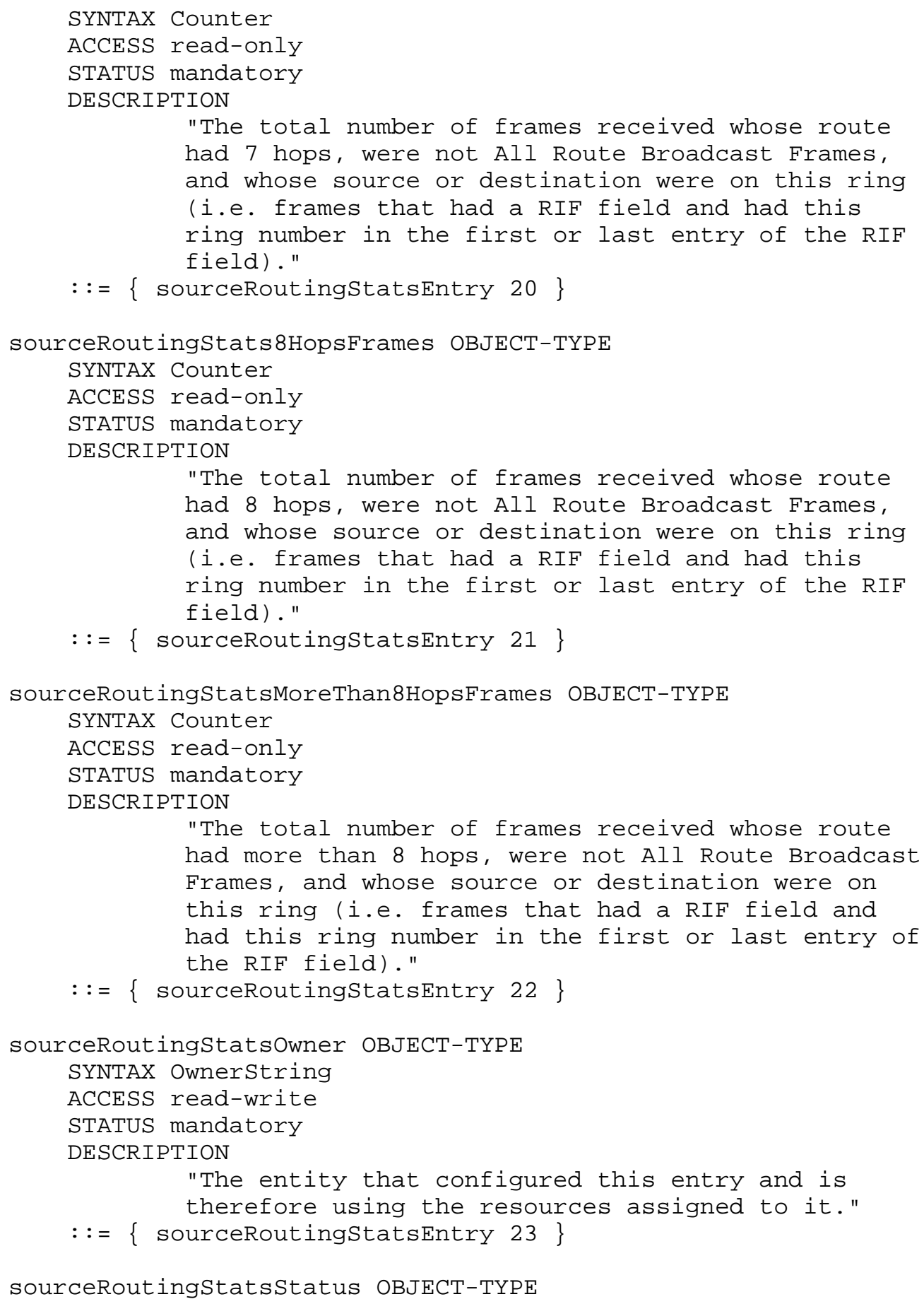




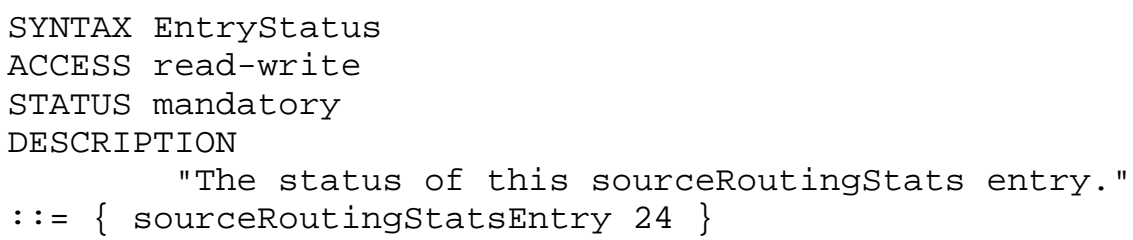

6. References

[1] Rose M., and K. MCCloghrie, "Structure and Identification of Management Information for TCP/IP-based internets", STD 16, RFC 1155, Performance Systems International, Hughes LAN Systems, May 1990 .

[2] Rose, M., and K. McCloghrie, Editors, "Concise MIB Definitions", STD 16, RFC 1212, Performance Systems International, Hughes LAN Systems, March 1991.

[3] McCloghrie K., and M. Rose, Editors, "Management Information Base for Network Management of TCP/IP-based internets", STD 17, RFC 1213, Performance Systems International, March 1991.

[4] Case, J., Fedor, M., Schoffstall, M., and J. Davin, "Simple Network Management Protocol", STD 15, RFC 1157, SNMP Research, Performance Systems International, Performance Systems International, MIT Laboratory for Computer Science, May 1990.

[5] Information processing systems - Open Systems Interconnection Specification of Abstract Syntax Notation One (ASN.1), International Organization for Standardization. International Standard 8824, December, 1987.

[6] Waldbusser, S., "Remote Network Monitoring Management Information Base", RFC 1271, CMU, November 1991.

[7] Token Ring Access Method and Physical Layer Specifications, Institute of Electrical and Electronic Engineers, IEEE Standard $802.5-1989,1989$. 
7. Acknowledgments

This document was produced by the Token Ring RMON MIB working group.

In addition, the author gratefully acknowledges the comments of the following individuals:

$\begin{array}{ll}\text { Andrew Bierman } & \text { Synoptics } \\ \text { Steve Bostock } & \text { Novell } \\ \text { Gary Ellis } & \text { Hewlett-Packard } \\ \text { Mike Erlinger } & \text { Aerospace Corporation } \\ \text { Robert Graham } & \text { Protools } \\ \text { Stephen Grau } & \text { Novell } \\ \text { Carl Hayssen } & \text { Ungermann-Bass } \\ \text { Jeff Hughes } & \text { Hewlett-Packard } \\ \text { Robin Iddon } & \text { AxoN Networks } \\ \text { Ken Kutzler } & \text { Synoptics } \\ \text { To-Choi Lau } & \text { Novell } \\ \text { Carl Madison } & \text { Startek } \\ \text { Keith McCloghrie } & \text { Hughes Lan Systems } \\ \text { Rohit Mital } & \text { Protools } \\ \text { Keith Schomburg } & \text { IBM } \\ \text { Marshall Rose } & \text { Dover Beach Consulting } \\ \text { Mark Therieau } & \text { Microcom } \\ \text { Mark van der Pol } & \text { Hughes Lan Systems } \\ \text { Brian Wyld } & \text { Consultant } \\ \end{array}$

8. Security Considerations

Security issues are not discussed in this memo.

9. Author's Address

Steven Waldbusser

Carnegie Mellon University

4910 Forbes Ave.

Pittsburgh, PA 15213

Phone: (412) 268-6628

EMail: waldbusser@ cmu.edu 